\title{
Weak antilocalization and localization in disordered and interacting Weyl semimetals
}

\author{
Hai-Zhou Lu ${ }^{1,2}$ and Shun-Qing Shen ${ }^{2}$ \\ ${ }^{1}$ Department of Physics, South University of Science and Technology of China, Shenzhen, China \\ ${ }^{2}$ Department of Physics, The University of Hong Kong, Pokfulam Road, Hong Kong, China
}

(Received 12 November 2014; published 7 July 2015)

\begin{abstract}
Using the Feynman diagram techniques, we derive the finite-temperature conductivity and magnetoconductivity formulas from the quantum interference and electron-electron interaction, for a three-dimensional disordered Weyl semimetal. For a single valley of Weyl fermions, we find that the magnetoconductivity is negative and proportional to the square root of magnetic field at low temperatures, as a result of the weak antilocalization. By including the contributions from the weak antilocalization, Berry curvature correction, and Lorentz force, we compare the calculated magnetoconductivity with a recent experiment. The weak antilocalization always dominates the magnetoconductivity near zero field, thus gives one of the transport signatures for Weyl semimetals. In the presence of strong intervalley scattering and correlations, we expect a crossover from the weak antilocalization to weak localization. In addition, we find that the interplay of electron-electron interaction and disorder scattering always dominates the conductivity at low temperatures and leads to a tendency to localization. Finally, we present a systematic comparison of the transport properties of single-valley Weyl fermions, 2D massless Dirac fermions, and $3 \mathrm{D}$ conventional electrons.
\end{abstract}

DOI: 10.1103/PhysRevB.92.035203

PACS number(s): 72.25.-b, 75.47.-m, 78.40.Kc

\section{INTRODUCTION}

Weyl semimetal is a three-dimensional (3D) topological state of matter, in which the conduction and valence energy bands touch at a finite number of nodes [1]. The nodes always appear in pairs, in each pair the quasiparticles (dubbed Weyl fermions) carry opposite chirality and linear dispersion, much like a 3D analog of graphene. The neutrino used to be a potential candidate for the Weyl fermion, until its tiny mass was revealed. In the past few years, a number of condensed matter systems have been suggested to host Weyl fermions [2-13]. Most recently, the signatures of Weyl nodes have been observed by angle-resolved photoemission spectroscopy, scanning tunneling microscopy, and time-domain terahertz spectroscopy in $\left(\mathrm{Bi}_{1-x} \mathrm{In}_{x}\right)_{2} \mathrm{Se}_{3}[14,15], \mathrm{Na}_{3} \mathrm{Bi}[16,17], \mathrm{Cd}_{3} \mathrm{As}_{2}$ [18-22], and TlBiSSe [23]. (Strictly speaking, they are Dirac semimetals in which the paired Weyl nodes are degenerate [7,24].)

Excellent electronic transport is anticipated in Weyl semimetals. The Weyl nodes remain gapless unless being annihilated in pairs. It is known that disorder may induce a semimetal to metal transition [25-28]. Nevertheless, metals may also exhibit "insulating" behaviors as a result of disorder and quantum interference, i.e., Anderson localization [29]. In contrast, because of the symplectic symmetry [30,31] near each Weyl node, the Weyl fermions are immune from Anderson localization and tend to be "antilocalized," in the absence of interaction and intervalley scattering. One of the signatures of the weak antilocalization is a negative magnetoconductivity, and has been observed recently in $\mathrm{Bi}_{0.97} \mathrm{Sb}_{0.03}$ [32] with a theoretical description based on a corrected semiclassical Boltzmann equation [33], $\mathrm{ZrTe}_{5}$ [34], and TaAs [35,36]. However, to include the weak (anti)localization corrections, higher-order Feynman diagrams [29,37-39] beyond the semiclassical transport theory have to be taken into account. A full three-dimensional calculation beyond the semiclassical [24,33,40,41] and quasi-two-dimensional [42] regimes is still lacking for this paradigmatic system, in particular in the presence of many-body interaction and multivalley effects.

In this work, we systematically study the temperature and magnetic field dependencies of the conductivity of a two-valley Weyl semimetal. With the help of Feynman diagram techniques, we take into account high-order corrections from the quantum interference as well as the interplay of interaction and disorder beyond the semiclassical transport theory. We find that the low-temperature magnetoconductivity is negative and follows a square-root law in weak magnetic fields $B$, (i.e., a $-\sqrt{B}$ magnetoconductivity) (see Fig. 3) arising from the weak antilocalization, which is in consistence with the experiments [32,34-36]. However, despite this magnetoconductivity signature of the weak antilocalization, the temperature dependence of the conductivity still shows a tendency to localization below a critical temperature, as a result of weak many-body interaction (see Fig. 2). Moreover, intervalley scattering and correlation may also strengthen the localization tendency (see Fig. 3). This work brings the transport theory to the level of relevant experiments to detect signatures of Weyl fermions in solid-state systems.

The paper is organized as follows. In Sec. II, we introduce the model that describes a two-valley Weyl semimetal in the presence of electron-electron interaction and disorder. Then we briefly present the Feynman diagrams for the conductivity. In Sec. III, we show the temperature dependence of the conductivity at low temperatures. We focus on the competition between the weak antilocalization due to the quantum interference and the localization arising from the interplay of interaction and disorder scattering. In Sec. IV, we present the $-\sqrt{B}$ magnetoconductivity from the weak antilocalization of a single valley of Weyl fermions. Then we discuss the crossover to the weak localization as a result of the intervalley scattering and correlation. We also compare with a recent experiment, by including the magnetoconductivity contributions from the weak antilocalization, Berry curvature correction, and Lorentz force. In Sec. V, we compare the 
transport properties for 3D Weyl fermions, 2D massless Dirac fermions, and 3D conventional electrons. From Secs. VI through $\mathrm{X}$, we present detailed calculations for different contributions to the conductivity and magnetoconductivity.

\section{MODEL AND METHOD}

One of the low-energy descriptions of the interacting Weyl semimetal is

$$
H=\sum_{\mathbf{k}, v} \psi_{\mathbf{k} v}^{\dagger}\left[v \hbar v_{F} \boldsymbol{\sigma} \cdot\left(\mathbf{k}+v \mathbf{k}_{c}\right)\right] \psi_{\mathbf{k} v}+\sum_{\mathbf{q}} \frac{V_{\mathbf{q}}}{2} \hat{\rho}_{\mathbf{q}} \hat{\rho}_{-\mathbf{q}},
$$

where $\psi_{\mathbf{k} v}^{\dagger}=\left(\psi_{\mathbf{k} v \uparrow}^{\dagger}, \psi_{\mathbf{k} v \downarrow}^{\dagger}\right)$ is a two-component spinor operator with the valley index $v= \pm$ describing the opposite chirality and $\uparrow / \downarrow$ for the spin index. The corresponding density operator is $\hat{\rho}_{\mathbf{q}}=\sum_{v, \mathbf{k}} \psi_{\mathbf{k} v}^{\dagger} \psi_{\mathbf{k}+\mathbf{q}, v} \cdot v_{F}$ is the Fermi velocity, $\hbar$ is the reduced Planck constant, $\sigma=\left(\sigma_{x}, \sigma_{y}, \sigma_{z}\right)$ is the vector of Pauli matrices, and $\pm \mathbf{k}_{c}$ are the two Weyl nodes. In international unit $V_{\mathbf{q}}=e^{2} / \varepsilon q^{2}$ in $3 \mathrm{D}$, with $\varepsilon$ the dielectric constant. In realistic materials, Weyl fermions are also perturbed by disorder $U(\mathbf{r})$. For mathematical convenience, we assume the delta potential $U(\mathbf{r})=\sum_{i} u_{i} \delta\left(\mathbf{r}-\mathbf{R}_{i}\right)$, where $u_{i}$ measures the random potential at position $\mathbf{R}_{i}$, and delta correlation between the impurities, $\left\langle U(\mathbf{r}) U\left(\mathbf{r}^{\prime}\right)\right\rangle \sim \delta\left(\mathbf{r}-\mathbf{r}^{\prime}\right)$.

We employ the Feynman diagram techniques to calculate the conductivity in the presence of disorder and interaction (see Fig. 1). In this theoretical framework, the conductivity includes three dominant parts, the semiclassical (Drude) conductivity [40,41] $\sigma^{s c}$ [see Fig. 1(a)], the correction from the quantum interference $\sigma^{q i}$ [see Fig. 1(b)], and the correction from the interplay of electron-electron interaction and disorder scattering $\sigma^{e e}$ [see Fig. 1(c)]. We will first focus on one valley, then move on to the multivalley case. Along an arbitrary measurement direction, the Drude conductivity is found as $\sigma^{s c}=e^{2} N_{F} D$ (see Sec. VII for the calculation), which satisfies the Einstein relation. The density of states at the Fermi energy $E_{F}$ per valley $N_{F}=E_{F}^{2} / 2 \pi^{2}\left(v_{F} \hbar\right)^{3}$, the diffusion coefficient

(a) $\sigma^{s c}$

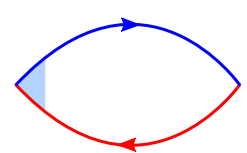

(b) $\sigma^{q i}$

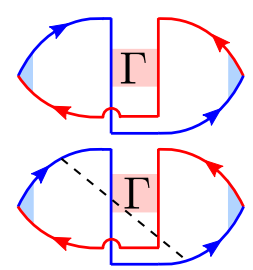

(c) $\sigma^{e e}$
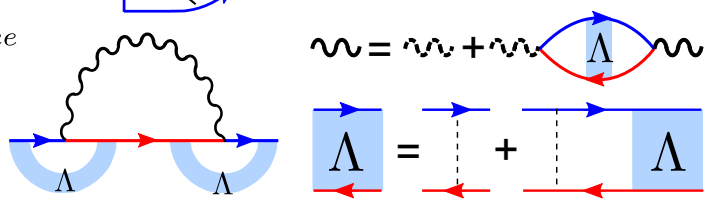

FIG. 1. (Color online) The Feynman diagrams [29,37-39,43-45] for the conductivity of 3D Weyl semimetals, in the presence of disorder (dashed lines) and electron-electron interaction (wavy lines). The arrow lines are for Green's functions.
$D=v_{F}^{2} \tau \eta_{v} / 3$, with $\tau$ the total momentum relaxation time and the correction to velocity by the ladder diagrams $\eta_{v}=3 / 2$ $[40,42]$. We find that intervalley scattering can modify $\eta_{v}$ to $(3 / 2) /\left(1+\eta_{I}\right)$, where $\eta_{I} \in[0,1]$ measures the weight of the intervalley scattering in the total scattering.

\section{FINITE-TEMPERATURE CONDUCTIVITY}

\section{A. Quantum interference and weak antilocalization}

According to the classification of random ensembles [46], systems with time-reversal symmetry but broken spinrotational symmetry are classified into the symplectic class. A symplectic system is supposed to exhibit the weak antilocalization effect [30], when the quantum interference [Fig. 1(b)] corrects the conductivity. Weyl fermions in a single valley have the symplectic symmetry so the weak antilocalization effect is expected. We find that the quantum interference correction for one valley of Weyl fermions takes the form (detailed calculation in Sec. VIII)

$$
\sigma^{q i}(T)=\frac{e^{2}}{h} \frac{1}{\pi^{2}}\left(\frac{1}{\ell}-\frac{1}{\ell_{\phi}}\right),
$$

where $e^{2} / h$ is the conductance quantum, $\ell$ is the mean free path, and $\ell_{\phi}$ is the phase coherence length. This single-valley result has exactly the same magnitude but opposite sign compared to that for conventional 3D electrons [with dispersion $(\hbar k)^{2} / 2 m$ ] per spin [29]. With decreasing temperature, $\ell_{\phi}$ always increases as decoherence induced by inelastic scattering is suppressed gradually. Therefore $\sigma^{q i}$ will be enhanced when lowering the temperature, literally giving a weak antilocalization contribution (see $\sigma^{q i}$ in Fig. 2). The temperature dependence of $\sigma^{q i}$ is from $\ell_{\phi}=C T^{-p / 2}$ [47], where $C$ is a constant and $p$ depends only on dimensionality and decoherence mechanisms thus does not distinguish conventional systems and Weyl semimetals. In $3 \mathrm{D}, p=3 / 2$ $(p=3)$ if electron-electron (electron-phonon) interaction is the decoherence mechanism in the disordered limit [29]. Also, because our calculation is in $3 \mathrm{D}$, the functional relationship is not logarithmic as that in quasi-2D $[42,48]$. For Weyl
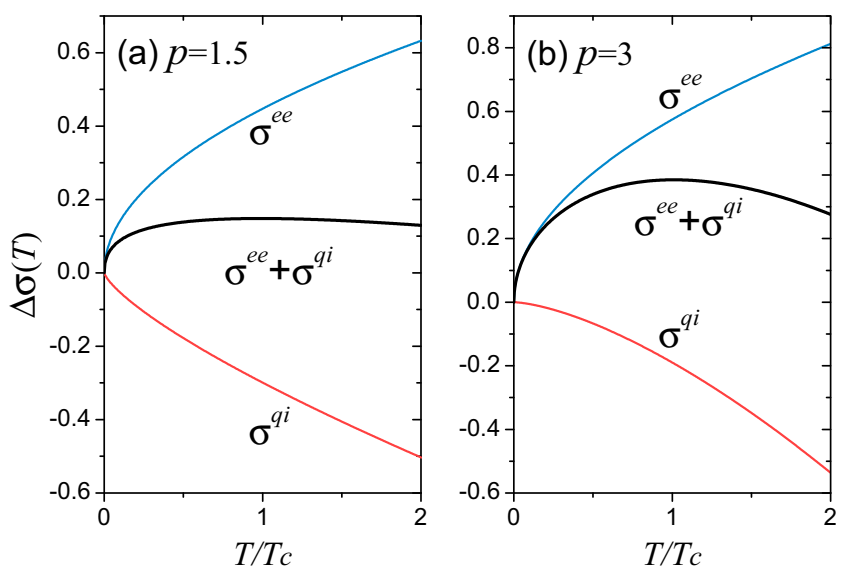

FIG. 2. (Color online) A schematic demonstration of the change of conductivity $\Delta \sigma$ as a function of temperature $T$. We choose $c_{e e}=$ $c_{q i} . T_{c}$ is the critical temperature below which the conductivity drops with decreasing temperature. 
semimetals realized by breaking time-reversal symmetry [8], the weak antilocalization may be suppressed by magnetism.

\section{B. Weak localization induced by the interplay of interaction and disorder}

Despite the signature in magnetoconductivity, we will show that the weak antilocalization breaks down in the presence of many-body interactions. The dominant interaction correction to the conductivity is attributed to the one-loop Fock (exchange interaction) self-energy dressed by diffusons [Fig. 1(c)]. We find that, for Weyl fermions, this self-energy gives a correction of the same form as that for conventional 3D electrons, upon a redefinition of the parameters such as the diffusion coefficient $D$. Besides the self-energy in Fig. 1(c), there are three other one-loop self-energies (see Fig. 10). These four self-energies contribute to a correction to the conductivity:

$$
\sigma^{e e}(T) \approx \frac{e^{2}}{h}(1-F) \sqrt{\frac{k_{B} T}{\hbar D}} \times 0.195
$$

where $k_{B}$ is the Boltzmann constant and the screening factor $F$ is defined as the average of the interaction over the Fermi surface. In 3D, $F=[\ln (1+x)] / x$ [29], where we find $x=8 \pi^{2} v_{F} \hbar \varepsilon / e^{2}$ for Weyl fermions, with $\varepsilon$ the dielectric constant. By definition, $F \in[0,1]$, as shown in Table I for several popular candidates of Weyl semimetal. Therefore $\sigma^{e e}$ decreases with decreasing temperature following a law of $\sqrt{T}$, giving a localization tendency. Disorder is inevitable in realistic materials so here the interaction is dressed by disorder, while in the clean limit the interaction alone may give a linear- $T$ conductivity [24,27]. In the clean limit, a marginal Fermi liquid phase and generation of mass are found for Dirac semimetals [49]. Also, $F$ will be further corrected to $\widetilde{F}$ after including the second-order interaction self-energies and interaction correction to the disorder scattering [29]. However, $\widetilde{F} \sim F$ and $\widetilde{F} \leqslant F$ (see Table I and Fig. 13). Later, we will see that there is always a localization tendency as long as $1-F>0$, where the dominant 1 is contributed by the selfenergy in Fig. 1(c). The interaction part $\sigma^{e e}$ also contributes to a negative magnetoconductivity, with a magnitude much smaller than $\delta \sigma^{q i}(B)$, this property is consistent with conventional electrons [29].

TABLE I. The dielectric constant $\varepsilon$ (in units of vacuum dielectric constant), Fermi velocity $v_{F}$, and screening factor $F$ and $\widetilde{F}$ (after the renormalization) for several candidates of Weyl semimetals. Because of anisotropy, $v_{F} \hbar$ covers a wide range in $\mathrm{Bi}_{0.97} \mathrm{Sb}_{0.03}$ and $\mathrm{Cd}_{3} \mathrm{As}_{2}$.

\begin{tabular}{lcccc}
\hline \hline & $\varepsilon$ & $v_{F} \hbar[\mathrm{eV} \AA]$ & $F$ & $\widetilde{F}$ \\
\hline $\mathrm{Bi}_{0.97} \mathrm{Sb}_{0.03}$ & 100 & $1-10$ & $0.09-0.01$ & $0.09-0.01$ \\
Refs. & {$[50]$} & {$[51,52]$} & & \\
TlBiSSe & 20 & 1.1 & 0.25 & 0.24 \\
Refs. & {$[23]$} & {$[23]$} & & \\
$\mathrm{Cd}_{3} \mathrm{As}_{2}$ & $36-52$ & $2-7$ & $0.03-0.11$ & $0.03-0.11$ \\
Refs. & {$[53-55]$} & {$[11,21]$} & & \\
\hline \hline
\end{tabular}

\section{Competing weak antilocalization and localization}

Combining $\sigma^{q i}$ and $\sigma^{e e}$, the change of conductivity with temperature for one valley of Weyl fermions can be summarized as

$$
\Delta \sigma(T)=c_{e e} T^{1 / 2}-c_{q i} T^{p / 2},
$$

where $c_{e e}=0.195(1-F) \sqrt{k_{B} / \hbar D}$ and $c_{q i}=1 / c \pi^{2}$ in units of $e^{2} / h$. This describes a competition between the interactioninduced weak localization and interference-induced weak antilocalization, as shown in Fig. 2 schematically. At higher temperatures, the conductivity increases with decreasing temperature, showing a weak antilocalization behavior. Below a critical temperature $T_{c}$, the conductivity starts to drop with decreasing temperature, exhibiting a localization tendency. From $\partial \Delta \sigma / \partial T=0$, the critical temperature can be found as $T_{c}=\left(c_{e e} / p \cdot c_{q i}\right)^{2 /(p-1)}$, at which $\left.\left(\partial^{2} \Delta \sigma / \partial T^{2}\right)\right|_{T_{c}} \approx(1-$ $p)\left(p c_{q i} / 4\right)\left(c_{e e} / p c_{q i}\right)^{(p-4) /(p-1)}$. Because $c_{e e}, c_{q i}>0$, this means that as long as $p>1$, there is always a critical temperature below which the conductivity drops with decreasing temperature. For known decoherence mechanisms in $3 \mathrm{D}, p$ is always greater than 1 [29]. Now we estimate the critical temperature $T_{c}$. Using $c_{e e}$ and $c_{q i}$, we arrive at $T_{c} \approx\left[C(1-F) /\left(2 p \sqrt{v_{F} \ell}\right)\right]^{2 /(p-1)}$, which shows that $T_{c}$ increases with $C$ while decreases with $F, p, v_{F}$, and $\ell$. With a set of typical parameters $F=0.25 \sim 0.01$ and $v_{F}=$ $10 \sim 1 \times 10^{5} \mathrm{~m} / \mathrm{s}$, as well as $p=3 \sim 3 / 2, C=100 \sim$ $1000 \mathrm{~nm} \mathrm{~K}^{p / 2}, \ell=100 \sim 10 \mathrm{~nm}$ in disordered metals, we find that $T_{c} \approx 0.4 \sim 10^{6} \mathrm{~K}$. Please note that our calculation is not justified at high temperatures, but in this way we show that the localization tendency is experimentally accessible in disordered Weyl semimetals. For $\mathrm{Cd}_{3} \mathrm{As}_{2}$ with extremely high mobility, the recent experiment [56] demonstrated that the mean free path $\ell$ is well above $1 \mu \mathrm{m}$, yielding a $T_{c}$ well below those achievable $(10 \mathrm{mK})$ in most laboratories.

\section{MAGNETOCONDUCTIVITY}

\section{A. The $-\sqrt{B}$ magnetoconductivity of a single valley of Weyl fermions}

Because of its quantum interference origin, $\sigma^{q i}$ in Eq. (2) can be suppressed by a magnetic field, giving rise to a magnetoconductivity $\delta \sigma^{q i}(B) \equiv \sigma^{q i}(B)-\sigma^{q i}(0)$, where

$$
\begin{aligned}
\sigma^{q i}(B)= & \frac{2 e^{2}}{h} \int_{0}^{1 / \ell} \frac{d x}{(2 \pi)^{2}}\left[\psi\left(\frac{\ell_{B}^{2}}{\ell^{2}}+\ell_{B}^{2} x^{2}+\frac{1}{2}\right)\right. \\
& \left.-\psi\left(\frac{\ell_{B}^{2}}{\ell_{\phi}^{2}}+\ell_{B}^{2} x^{2}+\frac{1}{2}\right)\right]
\end{aligned}
$$

for one valley of Weyl fermions, with $\psi$ the digamma function and $\ell_{B} \equiv \sqrt{\hbar / 4 e B}$ the magnetic length. The magnetic field $B$ is applied along an arbitrary direction (we have checked that $\sigma_{x x}^{q i}=\sigma_{z z}^{q i}$ ). As $B \rightarrow \infty, \delta \sigma^{q i}$ saturates following a $1 / B$ dependence. As $B \rightarrow 0, \delta \sigma^{q i}$ is proportional to $-\sqrt{B}$ for $\ell_{\phi} \gg \ell_{B}$ or at low temperatures, and $\delta \sigma^{q i} \propto-B^{2}$ for $\ell_{\phi} \ll \ell_{B}$ at high temperatures. $\ell_{B}$ can be evaluated approximately as $12.8 \mathrm{~nm} / \sqrt{B}$ with $B$ in Tesla. Usually below the liquid helium temperature, $\ell_{\phi}$ can be as long as hundreds of nanometers to one micrometer, much longer than $\ell_{B}$, which is tens 

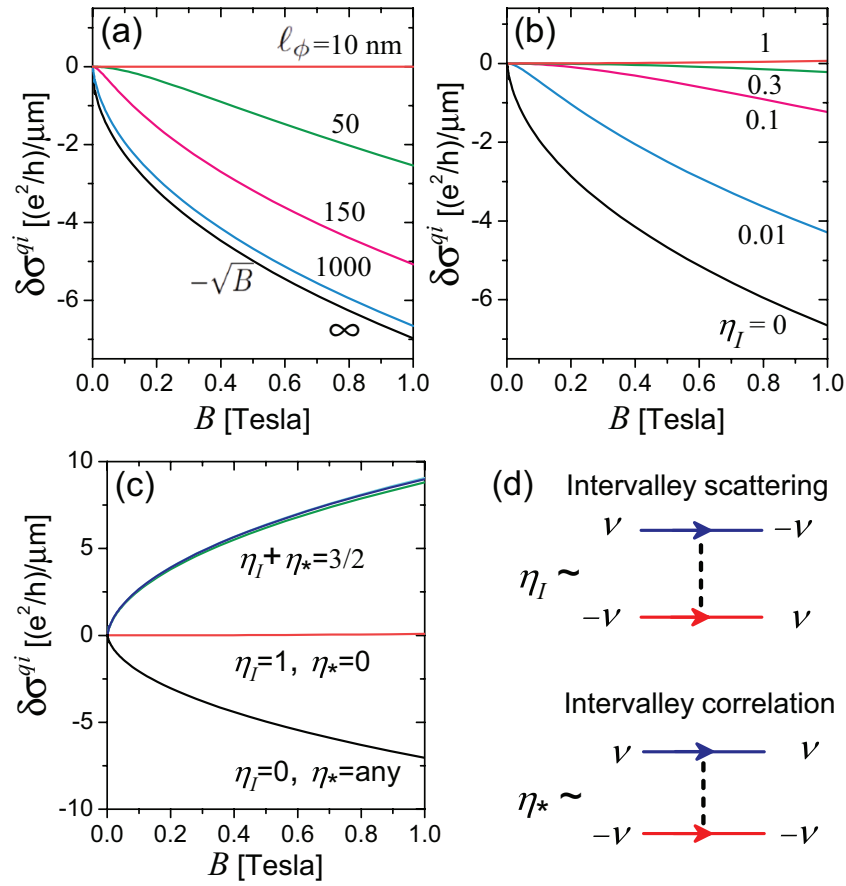

(d) Intervalley scattering

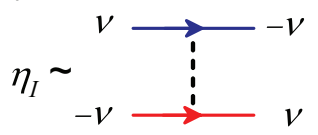

Intervalley correlation

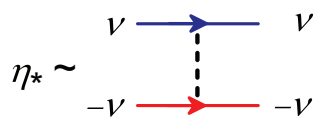

FIG. 3. (Color online) The magnetoconductivity $\delta \sigma^{q i}(B)$ for different phase coherence lengths $\ell_{\phi}$ at $\eta_{I}=\eta_{*}=0$ (a), for different $\eta_{I}$ at $\eta_{*}=0$ (b), and for different $\eta_{I}$ at finite $\eta_{*}$ (c). Parameters: $\ell=10 \mathrm{~nm}$ and $\ell_{\phi}=1000 \mathrm{~nm}$ in (b) and (c). (d) The diagrams show the difference between $\eta_{I}$ and $\eta_{*}$, with $\eta_{I}$ related to the intervalley scattering and $\eta_{*}$ measuring the intervalley correlation of intravalley scattering. The dashed lines represent the correlation of two scattering processes. $v= \pm$ is the valley index.

of nanometers between 0.1 and 1 T. Therefore the $-\sqrt{B}$ magnetoconductivity at low temperatures and small fields serves as a signature for the weak antilocalization of 3D Weyl fermions. Figure 3(a) shows $\delta \sigma^{q i}(B)$ of two valleys of Weyl fermions in the absence of intervalley scattering. For long $\ell_{\phi}, \delta \sigma^{q i}(B)$ is negative and proportional to $\sqrt{B}$, showing the signature of the weak antilocalization of $3 \mathrm{D}$ Weyl fermions. This $-\sqrt{B}$ dependence agrees well with the experiment [32,33], and we emphasize that it is obtained from a complete diagram calculation with only two parameters $\ell$ and $\ell_{\phi}$ of physical meaning. As $\ell_{\phi}$ becomes shorter, a change from $-\sqrt{B}$ to $-B^{2}$ is evident. $\delta^{q i}(B)$ vanishes at $\ell_{\phi}=\ell$ as the system quits the quantum interference regime. Also, it is known that the chiral anomaly could give a positive magnetoconductivity $[33,41,57,58]$, competing with the negative magnetoconductivity from the weak antilocalization. This chiral-anomaly part, because of its $B^{2}$ dependence, will always be overwhelmed by the $-\sqrt{B}$ weak antilocalization part at weak magnetic fields. At high fields, the chiral anomaly may become dominant.

\section{B. Weak localization induced by intervalley effects}

Now, we come to consider the effects of intervalley scattering and correlation. We will focus on the quantum interference part and magnetoconductivity [see Sec. VIIIC for the expressions of $\sigma^{q i}$ and $\delta \sigma^{q i}(B)$ in the presence of intervalley scattering and correlation], because we find that the interaction brings a negligible valley-dependent effect. Two dimensionless parameters are defined for the inter- and intravalley scattering: $\eta_{*} \propto\left\langle U_{\mathbf{k}, \mathbf{k}^{\prime}}^{++} U_{\mathbf{k}^{\prime}, \mathbf{k}}^{--}\right\rangle$measuring the correlation between intravalley scattering and $\eta_{I} \propto\left\langle U_{\mathbf{k}, \mathbf{k}^{\prime}}^{+-} U_{\mathbf{k}^{\prime}, \mathbf{k}}^{-+}\right\rangle$ measuring the weight of intervalley scattering, where $U_{\mathbf{k}, \mathbf{k}^{\prime}}^{v, v^{\prime}}$ is the scattering matrix element. Figure 3(d) schematically shows the difference between $\eta_{*}$ and $\eta_{I}$. As shown in Fig. 3(b), with increasing $\eta_{I}$, the negative $\delta \sigma^{q i}$ is suppressed, where $\eta_{I} \rightarrow$ 1 means strong intervalley scattering while $\eta_{I} \rightarrow 0$ means vanishing intervalley scattering. Furthermore, Fig. 3(c) shows that the magnetoconductivity can turn to positive when $\eta_{I}+$ $\eta_{*}=3 / 2$. Remember that the negative $\delta \sigma^{q i}(B)$ in Fig. 3(b) is related to the increasing $\sigma^{q i}(T)$ with decreasing $T$ in Fig. 2, as two signatures of the weak antilocalization. Similarly, the positive $\delta \sigma^{q i}(B)$ in Fig. 3(c) corresponds to a suppressed $\sigma^{q i}$ with decreasing temperature, i.e., a localization tendency. This localization is attributed to the strong intervalley coupling, which recovers spin-rotational symmetry (now the spin space is complete for a given momentum), then the system goes to the orthogonal class $[30,31,46]$. Therefore we show that the combination of strong intervalley scattering and correlation will strengthen the localization tendency in disordered Weyl semimetals.

\section{Comparison with magnetoconductivity in experiments}

To compare with experiments, besides the magnetoconductivity $\delta \sigma^{q i}(B)$ arising from the weak antilocalization in Sec. IV A, two more contributions to the total magnetoconductivity have to be taken into account. One is the classical negative magnetoconductivity due the cyclotron motion of electron driven by the Lorentz force in perpendicular magnetic fields and is given by $\delta \sigma^{C}=-\sigma^{s c} \mu^{2} B^{2}$ [59], where for the Weyl fermion the mobility is given by $\mu=e v_{F} \tau \eta_{v} / \hbar k_{F}$, then (see Sec. X for details)

$$
\delta \sigma^{C}(B)=-\frac{e^{2}}{h} \frac{\sqrt{3} \eta_{v}^{3 / 2}}{16 \pi} \frac{\ell^{3}}{\ell_{B}^{4}} .
$$

This part arises only in a perpendicular field and is not a function of $k_{F}$. It becomes dominant for long $\ell$, i.e., in highmobility and clean samples.

The other semiclassical magnetoconductivity is from the chiral anomaly, which arises because of the nontrivial Berry curvature carried by Weyl fermions, and it can give a magnetic field dependent correction to the velocity and Drude conductivity. An explicit form of $\delta \sigma^{A}(B)$ has been derived by Son and Spivak [57] and Burkov [58]. For example, according to Burkov [58],

$$
\delta \sigma^{A}(B)=\frac{e^{4} B^{2} \tau_{a}}{4 \pi^{4} g\left(E_{F}\right)},
$$

where $\hbar=1, g\left(E_{F}\right)=2 N_{F} . \tau_{a}$ is referred to as the axial relaxation time, which is supposed to be an independent parameter. Here, we use the intervalley scattering time for the axial relaxation time. In terms of the notations used in this work,

$$
\delta \sigma^{A}(B)=\frac{e^{2}}{h} \frac{\ell}{\ell_{B}^{4}} \frac{1}{k_{F}^{2}} \frac{\sqrt{2\left(1+\eta_{I}\right)}}{32 \pi \eta_{I}} .
$$


TABLE II. Comparison between the classical magnetoconductivity (MC) induced by the Lorentz force $\delta \sigma^{C}(B)$, semiclassical MC induced by the chiral anomaly $\delta \sigma^{A}(B)$ [57,58], and weak antilocalization MC induced by the quantum interference $\delta \sigma^{q i}(B)$, in their dependences on magnetic field $B$, temperature $T$, Fermi wave vector $k_{F}$, and mean free path $\ell$, in the limit $\eta_{I} \rightarrow 0$.

\begin{tabular}{lccc}
\hline \hline Dependence & $\delta \sigma^{C}$ & $\delta \sigma^{A}$ & $\delta \sigma^{q i}\left(\eta_{I} \rightarrow 0\right)$ \\
\hline$B$ & $-B^{2}$ & $B^{2}$ & $-\sqrt{B}$ \\
$B$ direction & $\perp$ & $\|$ & Any \\
$T$ & No & No & Suppressed with increasing $T$ \\
$k_{F}$ & No & $1 / k_{F}^{2}$ & No \\
$\ell$ & $\ell^{3}$ & $\ell$ & Decreases with increasing $\ell$ \\
$\eta_{I}$ & No & $1 / \eta_{I}$ & Suppressed with increasing $\eta_{I}$ \\
\hline \hline
\end{tabular}

Here, $\ell_{B} \equiv \sqrt{\hbar / 4 e B}$. The Berry curvature correction may also be the reason for some anomalous magnetoconductivity in topological insulators [60].

Including the three contributions, now the total magnetoconductivity is

$$
\delta \sigma_{\|}(B)=\delta \sigma^{q i}(B)+\delta \sigma^{A}(B),
$$

when the current is parallel to the magnetic field, and

$$
\delta \sigma_{\perp}(B)=\delta \sigma^{q i}(B)+\delta \sigma^{C}(B),
$$

when the current is perpendicular to the magnetic field.

In Table II, we compare these three different magnetoconductivity. Please note that, $\delta \sigma^{C}$ dominates in clean samples because it is proportional to $\ell^{3}$ while $\delta \sigma^{A}$ dominates near Weyl nodes because it is proportional to $1 / k_{F}^{2}$, and $\delta \sigma^{q i}$ appear only at low temperatures.

In Fig. 4, we use Eqs. (9) and (10) to reproduce the magnetoconductivity measured by Kim et al. in $\mathrm{Bi}_{0.03} \mathrm{Sb}_{0.97}$
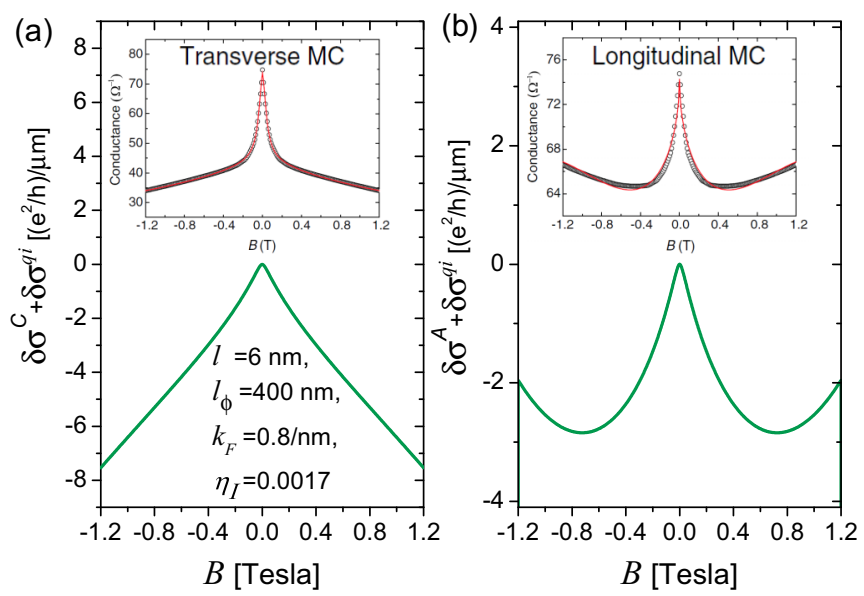

FIG. 4. (Color online) Comparison with the experimental magnetoconductivity by $\mathrm{Kim}$ et al. (insets) [32]. The transverse magnetoconductivity (MC) $\delta \sigma_{\perp}(B)=\delta \sigma^{C}+\delta \sigma^{q i}$ and longitudinal MC $\delta \sigma_{\|}(B)=\delta \sigma^{A}+\delta \sigma^{q i}$ are defined when the current is perpendicular and parallel to the magnetic field, respectively. The classical MC induced by the Lorentz force $\delta \sigma^{C}(B)$ is given in Eq. (6). The semiclassical MC induced by the chiral anomaly $\delta \sigma^{A}(B)[57,58]$ is in Eq. (8). The weak antilocalization MC induced by the quantum interference $\delta \sigma^{q i}(B)$ is in Sec. VIII C.
[32]. The main features (e.g., the transverse MC is several times of the longitudinal MC, those inflection points in MC) in both the transverse and longitudinal magnetoconductivity can be recovered simultaneously within a set of parameters comparable to those in relevant materials. Our parameters (e.g., mean free path, Fermi wave vector) are of physical meanings. Figure 4(a) is always negative because both $\delta \sigma^{C}$ and $\delta \sigma^{q i}$ are negative. The competition between $\delta \sigma^{q i} \propto-\sqrt{B}$ and $\delta \sigma^{A} \propto B^{2}$ leads to the inflection in Fig. 4(b).

\section{COMPARISON OF WEYL FERMIONS, 2D DIRAC FERMIONS, AND 3D CONVENTIONAL ELECTRONS}

To summarize, we compare the transport properties for a single valley of Weyl fermions, 2D massless Dirac fermions, and 3D conventional electrons, in Table III.

\section{THE CALCULATION OF THE CONDUCTIVITY}

Throughout the work, we will only focus on the conductivity of the conduction bands. The valence bands have the same properties. The eigenenergies of the conduction bands in the two valleys are degenerate:

$$
E_{\mathbf{k}}=v_{F} \hbar k=v_{F} \hbar \sqrt{k_{x}^{2}+k_{y}^{2}+k_{z}^{2}},
$$

where $\mathbf{k}$ is measured from each Weyl node. The spinor wave function of the conduction band in valley + is

$$
|+, \mathbf{k}\rangle=\left(\begin{array}{c}
\cos \frac{\theta}{2} \\
\sin \frac{\theta}{2} e^{i \varphi}
\end{array}\right) \frac{e^{i \mathbf{k} \cdot \mathbf{r}}}{\sqrt{\Omega}},
$$

where $\theta$ and $\varphi$ are the wave vector angles, $\tan \varphi \equiv k_{y} / k_{x}$, $\cos \theta \equiv k_{z} / k$, and $\Omega$ is the volume. In valley - , the wave function of the conduction band can be found as $(\theta \rightarrow \pi-\theta$ and $\varphi \rightarrow \pi+\varphi$ )

$$
|-, \mathbf{k}\rangle=\left(\begin{array}{c}
\sin \frac{\theta}{2} \\
-\cos \frac{\theta}{2} e^{i \varphi}
\end{array}\right) \frac{e^{i \mathbf{k} \cdot \mathbf{r}}}{\sqrt{\Omega}} .
$$

The total conductivity has three dominant parts:

$$
\sigma=\sigma^{s c}+\sigma^{q i}+\sigma^{e e} .
$$

$\sigma^{s c}$ is the semiclassical conductivity (Sec. VII), $\sigma^{q i}$ is the correction from the quantum interference (Sec. VIII), and $\sigma^{e e}$ is the correction from the interplay of electron-electron interaction and disorder scattering (Sec. IX).

\section{SEMICLASSICAL (DRUDE) CONDUCTIVITY}

The semiclassical (Drude) conductivity can be found as [Fig. 5(a)]

$$
\sigma^{s c}=\frac{e^{2} \hbar}{2 \pi} \sum_{\mathbf{k}} v_{\mathbf{k}}^{i} G_{\mathbf{k}}^{\mathrm{R}} G_{\mathbf{k}}^{\mathrm{A}} \widetilde{v}_{\mathbf{k}}^{i},
$$

where $i=x$ or $z, G^{R / A}$ is the retarded/advanced Green's function, $v_{\mathbf{k}}^{i}=(1 / \hbar) \partial E_{\mathbf{k}} / \partial k_{i}$ is the velocity, and $\widetilde{v}_{\mathbf{k}}^{i}$ is the corrected velocity by the disorder scattering [Fig. 5(b)]. The 
TABLE III. Comparison between a single valley of 2D massless Dirac fermions [31,37], a single valley of 3D Weyl fermions, and a single band of 3D conventional electrons [38,39]. $k_{F}$ is the Fermi wave vector, $m$ is the effective mass, $v_{F}$ is the constant Fermi velocity of Dirac and Weyl fermions, $\tau$ is the total momentum relaxation time, and $\eta_{v}$ is the correction to the velocity (Sec. VII). $\eta_{I} \in[0,1]$ measures the weight of intervalley scattering. $F$ is the screening factor of interaction (Sec. IX C). $\varepsilon$ is the dielectric constant and $\sigma^{e e}$ is the conductivity correction from the interplay of electron-electron interaction and disorder scattering. $\sigma^{q i}$ is the conductivity correction from the quantum interference. $\delta \sigma^{q i}(B)$ is the small-field magnetoconductivity from $\sigma^{q i}$ when $\ell_{\phi} \gg \ell_{B} . p$ is the exponent in the temperature dependence of the phase coherence length [47] $\ell_{\phi} \sim T^{-p / 2}$ due to electron-electron interaction (EEI) and electron-phonon (E-Ph) interaction in disordered metals [29].

\begin{tabular}{|c|c|c|c|}
\hline & 2D massless Dirac & 3D Weyl & 3D Conventional \\
\hline Dispersion $E(\mathbf{k})$ & $\pm \gamma \sqrt{k_{x}^{2}+k_{y}^{2}}$ & $\pm \gamma \sqrt{k_{x}^{2}+k_{y}^{2}+k_{z}^{2}}$ & $\left(\hbar^{2} / 2 m\right)\left(k_{x}^{2}+k_{y}^{2}+k_{z}^{2}\right)$ \\
\hline Density of states $N(E)$ & $E / 2 \pi\left(v_{F} \hbar\right)^{2}$ & $E^{2} / 2 \pi^{2}\left(v_{F} \hbar\right)^{3}$ & $(1 / 2 \pi)^{2}\left(2 m / \hbar^{2}\right)^{3 / 2} \sqrt{E}$ \\
\hline Carrier density per valley & $k_{F}^{2} / 4 \pi$ & $k_{F}^{3} / 6 \pi^{2}$ & $k_{F}^{3} / 6 \pi^{2}$ \\
\hline Mobility $\mu$ & $e v_{F} \tau \eta_{v} / \hbar k_{F}$ & $e v_{F} \tau \eta_{v} / \hbar k_{F}$ & $e \tau / m$ \\
\hline Diffusion coefficient $D$ & $v_{F}^{2} \tau \eta_{v} / 2$ & $v_{F}^{2} \tau \eta_{v} / 3$ & $\left(\hbar k_{F} / m\right)^{2} \tau / 3$ \\
\hline Velocity correction $\eta_{v}$ & $2[61]$ & $\begin{array}{c}3 / 2[40,42] \\
(3 / 2) /\left(1+\eta_{I}\right)\end{array}$ & 1 \\
\hline$\eta_{H}$ & $-1 / 4[37]$ & $-1 / 6[42]$ & 0 \\
\hline Screening factor $F$ & $\begin{array}{c}(2 / \pi)\left(\arctan \sqrt{1 / x^{2}-1}\right) / \sqrt{1-x^{2}} \\
x=8 \pi \varepsilon v_{F} \hbar / e^{2}\end{array}$ & $\begin{array}{c}{[\ln (1+x)] / x} \\
x=8 \pi^{2} \varepsilon v_{F} \hbar / e^{2}\end{array}$ & $\begin{array}{c}{[\ln (1+x)] / x} \\
x=8 \pi^{2} \varepsilon \hbar^{2} k_{F} / m e^{2}\end{array}$ \\
\hline$\delta \sigma^{q i}(B \rightarrow 0) \propto$ & $-B$ & $-\sqrt{B}$ & $\sqrt{B}[62]$ \\
\hline$\sigma^{e e}(T) \propto$ & $\ln T[45,63]$ & $\sqrt{T}$ & $\sqrt{T}[38,39]$ \\
\hline$\sigma^{q i}(T) \propto$ & $-\ln T[31,37]$ & $-T^{p / 2}$ & $T^{p / 2}[38,39]$ \\
\hline$p$ (EEI) [29] & 1 & $3 / 2$ & $3 / 2$ \\
\hline$p(\mathrm{E}-\mathrm{Ph})[29]$ & 3 & 3 & 3 \\
\hline
\end{tabular}

retarded $(\mathrm{R})$ and advanced (A) Green's functions

$$
G_{\mathbf{k}}^{\mathrm{R} / \mathrm{A}}(\omega)=\frac{1}{\omega-\xi_{\mathbf{k}} \pm i \frac{\hbar}{2 \tau}},
$$

where $\xi_{\mathbf{k}}=E_{\mathbf{k}}-E_{F}$. The total scattering time (or total momentum relaxation time) $\tau$ is defined as

$$
\frac{1}{\tau} \equiv \frac{1}{\tau_{0}}+\frac{1}{\tau_{I}},
$$

where the intra- and intervalley scattering times are given by

$$
\begin{aligned}
\frac{1}{\tau_{0}} & \equiv \frac{2 \pi}{\hbar} \sum_{\mathbf{k}^{\prime}}\left\langle\left|U_{\mathbf{k}, \mathbf{k}^{\prime}}^{++}\right|^{2}\right\rangle \delta\left(E_{F}-\xi_{\mathbf{k}^{\prime}}\right)=\frac{2 \pi}{\hbar} N_{F} \frac{n u_{0}^{2}}{2}, \\
\frac{1}{\tau_{I}} & \equiv \frac{2 \pi}{\hbar} \sum_{\mathbf{k}^{\prime}}\left\langle\left|U_{\mathbf{k}, \mathbf{k}^{\prime}}^{+-}\right|^{2}\right\rangle \delta\left(E_{F}-\xi_{\mathbf{k}^{\prime}}\right)=\frac{2 \pi}{\hbar} N_{F} \frac{n u_{I}^{2}}{2},
\end{aligned}
$$

$n$ is the impurity density, and $u_{0}$ and $u_{I}$ measure the strength for the intra- and intervalley scattering, respectively. $N_{F}=E_{F}^{2} / 2 \pi^{2}\left(v_{F} \hbar\right)^{3}$ is the density of states per valley. $U_{\mathbf{k}, \mathbf{k}^{\prime}}^{++} \equiv\left\langle+, \mathbf{k}|U(\mathbf{r})|+, \mathbf{k}^{\prime}\right\rangle$ and $U_{\mathbf{k}, \mathbf{k}^{\prime}}^{+-} \equiv\left\langle+, \mathbf{k}|U(\mathbf{r})|-, \mathbf{k}^{\prime}\right\rangle$ are the intravalley and intervalley scattering matrix elements, (a)

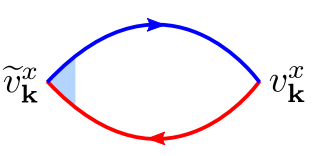

(b)

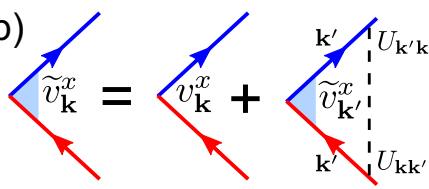

FIG. 5. (Color online) (a) The Feynman diagram for the semiclassical (Drude) conductivity $\sigma^{s c}$. (b) The diagram for the vertex correction to the velocity [61]. $v$ is the velocity. The arrow lines are for Green's functions. The dashed lines are for disorder scattering $(U)$. Replace $x$ by $z$ for the conductivity along the $z$ direction. respectively, and

$$
\begin{aligned}
& U_{\mathbf{k}, \mathbf{k}^{\prime}}^{++}=\sum_{i} u_{i} e^{i\left(\mathbf{k}^{\prime}-\mathbf{k}\right) \cdot \mathbf{R}_{i}}\left[a a^{\prime}+b b^{\prime} e^{i\left(\varphi^{\prime}-\varphi\right)}\right], \\
& U_{\mathbf{k}, \mathbf{k}^{\prime}}^{+-}=\sum_{i} u_{i} e^{i\left(\mathbf{k}^{\prime}-\mathbf{k}\right) \cdot \mathbf{R}_{i}}\left[a b^{\prime}-b a^{\prime} e^{i\left(\varphi^{\prime}-\varphi\right)}\right],
\end{aligned}
$$

with $a \equiv \cos (\theta / 2)$ and $b \equiv \sin (\theta / 2)$.

The correction to the velocity can be found from the iteration equation [Fig. 5(b)]

$$
\widetilde{v}_{\mathbf{k}}^{i}=v_{\mathbf{k}}^{i}+\sum_{\mathbf{k}^{\prime}} G_{\mathbf{k}^{\prime}}^{\mathrm{R}} G_{\mathbf{k}^{\prime}}^{\mathrm{A}}\left\langle U_{\mathbf{k}, \mathbf{k}^{\prime}} U_{\mathbf{k}^{\prime}, \mathbf{k}}\right\rangle \widetilde{v}_{\mathbf{k}^{\prime}}^{i} .
$$

In polar coordinates, $v_{\mathbf{k}}^{x}=v_{F} \sin \theta \cos \varphi$ and $v_{\mathbf{k}}^{z}=v_{F} \cos \theta$,

$$
\int_{0}^{\infty}\left(k^{\prime}\right)^{2} \frac{d k^{\prime}}{2 \pi} G_{\mathbf{k}^{\prime}}^{\mathrm{R}} G_{\mathbf{k}^{\prime}}^{\mathrm{A}} \approx \frac{2 \pi^{2} N_{F} \tau}{\hbar},
$$

and

$$
\begin{aligned}
\left\langle U_{\mathbf{k}, \mathbf{k}^{\prime}} U_{\mathbf{k}^{\prime}, \mathbf{k}}\right\rangle= & \left\langle U_{\mathbf{k}, \mathbf{k}^{\prime}}^{++} U_{\mathbf{k}^{\prime}, \mathbf{k}}^{++}\right\rangle+\left\langle U_{\mathbf{k}, \mathbf{k}^{\prime}}^{+-} U_{\mathbf{k}^{\prime}, \mathbf{k}}^{-+}\right\rangle \\
\approx & \frac{\hbar}{2 \pi N_{F} \tau}\left[1+\left(1-2 \eta_{I}\right)\right. \\
& \left.\times\left(\cos \theta \cos \theta^{\prime}+\sin \theta \sin \theta^{\prime} \cos \left(\varphi-\varphi^{\prime}\right)\right)\right],
\end{aligned}
$$

where $\eta_{I} \equiv \tau / \tau_{I}$ and $1 / \tau \equiv 1 / \tau_{0}+1 / \tau_{I}$, and $\tau_{0}$ and $\tau_{I}$ are the intravalley and intervalley scattering times, respectively. So $\eta_{I} \in[0,1]$ measures the relative strength of intervalley scattering. By assuming $\widetilde{v}_{\mathbf{k}}^{x}=\eta_{v} v_{\mathbf{k}}^{x}$ and $\widetilde{v}_{\mathbf{k}}^{z}=\eta_{v} v_{\mathbf{k}}^{z}$, and put them into the iteration equation for the velocity, one can readily find that for either the velocity along $x$ or $z$ direction,

$$
\eta_{v}=\frac{3}{2\left(1+\eta_{I}\right)} \text {. }
$$


Finally, we found that for either $x$ or $z$ direction,

$$
\sigma^{s c}=e^{2} N_{F} \frac{1}{3} v_{F}^{2} \tau \eta_{v},
$$

where the density of states per valley $N_{F}=E_{F}^{2} / 2 \pi^{2}\left(v_{F} \hbar\right)^{3}$; it satisfies the Einstein relation

$$
\sigma^{s c}=e^{2} N_{F} D,
$$

with the diffusion coefficient $D \equiv v_{F}^{2} \tau \eta_{v} / d$, where $d=3$ for three dimensions. Usually, $\tau \eta_{v}$ is referred to as the transport time. Later, we will show that $D$ can also be derived from the calculation of the diffuson (Sec. IX A).

In terms of the mean free path $\ell \equiv \sqrt{D \tau}$ and Fermi wave vector $k_{F}$,

$$
\sigma^{s c}=\frac{e^{2}}{h} \frac{k_{F}^{2} \ell}{\pi} \sqrt{\frac{\eta_{v}}{3}}=\frac{e^{2}}{h} \frac{k_{F}^{2} \ell}{\pi \sqrt{2\left(1+\eta_{I}\right)}} .
$$

\section{CONDUCTIVITY CORRECTION FROM QUANTUM INTERFERENCE}

The total conductivity from the quantum interference has two parts:

$$
\sigma^{q i}=2 \times \sigma_{0}^{q i}+\sigma_{I}^{q i} .
$$

$\sigma_{0}^{q i}$ is from the intravalley cooperons (Sec. VIII A) and $\sigma_{I}^{q i}$ is from the intervalley cooperons (Sec. VIII B).

\section{A. Conductivity correction from intravalley cooperons}

The conductivity contribution from the intravalley cooperons is given by (Fig. 6)

$$
\sigma_{0}^{q i}=\sigma_{a 1}+2 \sigma_{a 2}
$$

where

$$
\begin{aligned}
\sigma_{a 1}= & \frac{e^{2} \hbar}{2 \pi} \sum_{\mathbf{q}} \Gamma_{\mathbf{k}, \mathbf{q}-\mathbf{k}} \sum_{\mathbf{k}} G_{\mathbf{k}}^{R} \widetilde{v}_{\mathbf{k}}^{x} G_{\mathbf{k}}^{A} G_{\mathbf{q}-\mathbf{k}}^{R} \widetilde{v}_{\mathbf{q}-\mathbf{k}}^{x} G_{\mathbf{q}-\mathbf{k}}^{A}, \\
\sigma_{a 2}= & \frac{e^{2} \hbar}{2 \pi} \sum_{\mathbf{q}} \Gamma_{\mathbf{k}_{1}, \mathbf{q}-\mathbf{k}} \sum_{\mathbf{k}} \sum_{\mathbf{k}_{1}} \widetilde{v}_{\mathbf{k}}^{x} \widetilde{v}_{\mathbf{q}-\mathbf{k}_{1}}^{x} G_{\mathbf{k}}^{R} G_{\mathbf{k}_{1}}^{R} G_{\mathbf{q}-\mathbf{k}}^{R} \\
& \times G_{\mathbf{q}-\mathbf{k}_{1}}^{R} G_{\mathbf{k}}^{A} G_{\mathbf{q}-\mathbf{k}_{1}}^{A}\left\langle U_{\mathbf{k}, \mathbf{k}_{1}} U_{\mathbf{q}-\mathbf{k}, \mathbf{q}-\mathbf{k}_{1}}\right\rangle .
\end{aligned}
$$

We also find that the ratio of the dressed to bare Hikami boxes is

$$
\eta_{H} \equiv \frac{\sigma_{a 2}}{\sigma_{a 1}}=-\frac{1}{6},
$$

consistent with that by Garate and Glazman [42].

(a1)

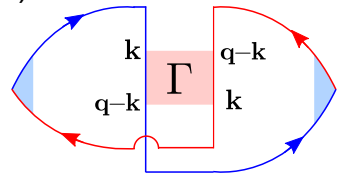

FIG. 6. (Color online) The Feynman diagrams for the quantum interference correction to the conductivity that take into account the cooperons from only the intravalley scattering. These diagrams give $\sigma_{0}^{q i}$.

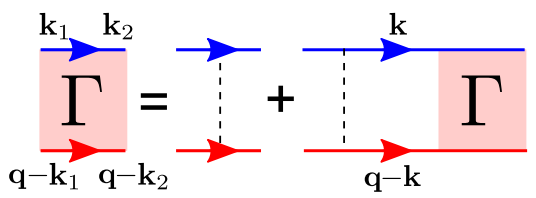

FIG. 7. (Color online) The Feynman diagram of the BetheSalpeter equation for the intravalley cooperons. The valley index is suppressed because the valley is conserved during the scattering.

In polar coordinates, the intravalley cooperon can be found by the Bethe-Salpeter equation (Fig. 7)

$$
\begin{aligned}
\Gamma_{\mathbf{k}_{1}, \mathbf{k}_{2}}= & \Gamma_{\mathbf{k}_{1}, \mathbf{k}_{2}}^{0}+\int_{0}^{2 \pi} \frac{d \varphi}{2 \pi} \int_{0}^{\pi} \frac{d \theta \sin \theta}{2 \pi} \int_{0}^{\infty} \frac{d k k^{2}}{2 \pi}, \\
& \times \Gamma_{\mathbf{k}_{1}, \mathbf{k}}^{0} \mathcal{G}_{\mathbf{k}}^{i \epsilon_{n}} \mathcal{G}_{\mathbf{q}-\mathbf{k}}^{i \epsilon_{n}-i \omega_{m}} \Gamma_{\mathbf{k}, \mathbf{k}_{2}},
\end{aligned}
$$

where the Matsubara Green's function is given as

$$
\mathcal{G}\left(\mathbf{k}, i \epsilon_{n}\right)=\frac{1}{i \hbar \epsilon_{n}-\xi_{\mathbf{k}}+i \frac{\hbar}{2 \tau} \operatorname{sgn}\left(\epsilon_{n}\right)},
$$

the fermionic Matsubara frequency $\epsilon_{n}=(2 n+1) \pi k_{B} T / \hbar$ with $n=0, \pm 1, \pm 2, \ldots$, the bosonic Matsubara frequency $\omega_{m}=2 \pi m k_{B} T / \hbar$ with $m=0, \pm 1, \pm 2, \ldots$, and $\xi_{\mathbf{k}}=E_{\mathbf{k}}-$ $E_{F}$ with $E_{F}$ the Fermi energy. The bare cooperon

$$
\begin{aligned}
\Gamma_{\mathbf{k}_{1}, \mathbf{k}_{2}}^{0} \equiv & \left\langle U_{\mathbf{k}_{1}, \mathbf{k}_{2}} U_{-\mathbf{k}_{1},-\mathbf{k}_{2}}\right\rangle \\
\approx & \frac{\hbar\left(1-\eta_{I}\right)}{2 \pi N_{F} \tau}\left[\frac{1}{2} \sin \theta_{1} \sin \theta_{2}+e^{i\left(\varphi_{2}-\varphi_{1}\right)}\right. \\
& \left.+\cos \theta_{1} \cos \theta_{2} e^{i\left(\varphi_{2}-\varphi_{1}\right)}+\frac{1}{2} \sin \theta_{1} \sin \theta_{2} e^{i 2\left(\varphi_{2}-\varphi_{1}\right)}\right],
\end{aligned}
$$

where $\eta_{I} \equiv \tau / \tau_{I}$ measures the relative strength of the intervalley scattering, and it can be found that

$$
\begin{aligned}
& \int_{0}^{\infty} \frac{d k k^{2}}{2 \pi} \mathcal{G}_{\mathbf{k}}^{i \epsilon_{n}} \mathcal{G}_{\mathbf{q}-\mathbf{k}}^{i \epsilon_{n}-i \omega_{m}} \\
& \quad \approx \frac{2 \pi^{2} N_{F} \tau}{\hbar} \frac{1}{1+\omega_{m} \tau+i \tau \mathbf{v}_{F} \cdot \mathbf{q}} \\
& \quad \approx 1-\omega_{m} \tau-i \tau v_{F} q \cos \theta-\tau^{2} v_{F}^{2} q^{2} \cos ^{2} \theta
\end{aligned}
$$

where $q^{2}=q_{x}^{2}+q_{y}^{2}+q_{z}^{2}$, which is essentially different from Ref. [42], where $q_{z}=0$ in a thin film with thickness $W \ll \ell_{\phi}$. We can assume the form of the intravalley cooperon

$$
\begin{aligned}
\Gamma_{\mathbf{k}_{1}, \mathbf{k}_{2}}= & \frac{\hbar}{2 \pi N_{F} \tau}\left[c_{1} \sin \theta_{1} \sin \theta_{2}+c_{2} e^{i\left(\varphi_{2}-\varphi_{1}\right)}\right. \\
& +c_{3} \cos \theta_{1} \cos \theta_{2} e^{i\left(\varphi_{2}-\varphi_{1}\right)}+c_{4} \sin \theta_{1} \sin \theta_{2} e^{i 2\left(\varphi_{2}-\varphi_{1}\right)} \\
& \left.+c_{5} \cos \theta_{1} e^{i\left(\varphi_{2}-\varphi_{1}\right)}+c_{6} \cos \theta_{2} e^{i\left(\varphi_{2}-\varphi_{1}\right)}\right] .
\end{aligned}
$$

By putting it into the Bethe-Salpeter equation, we can find that only the $c_{2}$ term is divergent as $\omega_{m}, q \rightarrow 0$ and

$$
\Gamma_{\mathbf{k}_{1}, \mathbf{k}_{2}} \approx \frac{\hbar}{2 \pi N_{F} D \tau^{2}} \frac{2+\eta_{I}}{2} \frac{e^{i\left(\varphi_{2}-\varphi_{1}\right)}}{q^{2}+Q_{0}^{2}},
$$


(c1)

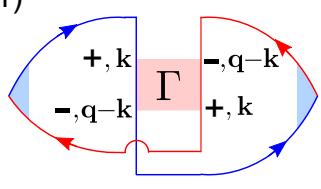

(c2)

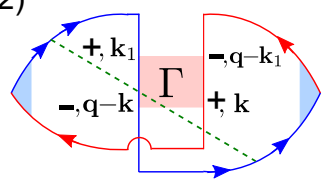

(d2)

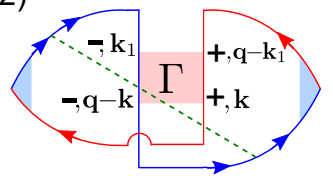

FIG. 8. (Color online) The Feynman diagrams for the quantum interference correction to the conductivity that take into account the cooperons from the intervalley scattering [64]. These diagrams give $\sigma_{I}^{q i}$.

where

$$
Q_{0}^{2} \equiv \frac{\left(2+\eta_{I}\right) \eta_{I}}{2\left(1-\eta_{I}\right)} \frac{1}{\ell^{2}}
$$

As $\eta_{I} \rightarrow 0$,

$$
\Gamma_{\mathbf{k}_{1}, \mathbf{k}_{2}}=\frac{\hbar}{2 \pi N_{F} D \tau^{2}} \frac{1}{q^{2}} e^{i\left(\varphi_{2}-\varphi_{1}\right)} .
$$

In the bare Hikami box, $\mathbf{k}_{1}=\mathbf{k}$ and $\mathbf{k}_{2}=\mathbf{q}-\mathbf{k} \approx-\mathbf{k}$, then $\varphi_{2}=\pi+\varphi_{1}, e^{i\left(\varphi_{2}-\varphi_{1}\right)}=e^{i \pi}=-1$. Similarly, in the dressed Hikami box, $e^{i\left(\varphi_{2}-\varphi_{1}\right)}$ becomes $e^{i\left(\varphi-\varphi_{1}\right)}$.

\section{B. Conductivity correction from intervalley cooperons}

The conductivity contribution from the intervalley cooperons is given by (Fig. 8)

$$
\sigma_{I}^{q i}=2\left(\sigma_{c 1}+2 \sigma_{c 2}+2 \sigma_{d 2}\right),
$$

where

$$
\begin{aligned}
\sigma_{c 1}= & \frac{e^{2} \hbar}{2 \pi} \sum_{\mathbf{q}} \Gamma_{-+}^{+-}(\mathbf{k},-\mathbf{k}) \\
& \times \sum_{\mathbf{k}} \widetilde{v}_{\mathbf{k},+}^{x} G_{\mathbf{k},+}^{R} G_{\mathbf{q}-\mathbf{k},-}^{R} \widetilde{v}_{\mathbf{q}-\mathbf{k},-}^{x} G_{\mathbf{q}-\mathbf{k},-}^{A} G_{\mathbf{k},+}^{A}, \\
\sigma_{c 2}= & \frac{e^{2} \hbar}{2 \pi} \sum_{\mathbf{q}} \Gamma_{-+}^{+-}\left(\mathbf{k}_{1},-\mathbf{k}\right) \\
& \times \sum_{\mathbf{k}} \sum_{\mathbf{k}_{1}} \widetilde{v}_{\mathbf{k},+}^{x} G_{\mathbf{k},+}^{R} G_{\mathbf{k}_{1},+}^{R} G_{\mathbf{q}-\mathbf{k},-}^{R} G_{\mathbf{q}-\mathbf{k}_{1},-}^{R} \\
& \times \widetilde{v}_{\mathbf{q}-\mathbf{k}_{1},-}^{x} G_{\mathbf{q}-\mathbf{k}_{1},-}^{A} G_{\mathbf{k},+}^{A}\left\langle U_{\mathbf{k}, \mathbf{k}_{1}}^{++} U_{\mathbf{q}-\mathbf{k}, \mathbf{q}-\mathbf{k}_{1}}^{--}\right\rangle, \\
\sigma_{d 2}= & \frac{e^{2} \hbar}{2 \pi} \sum_{\mathbf{q}} \Gamma_{++}^{--}\left(\mathbf{k}_{1},-\mathbf{k}\right) \\
& \times \sum_{\mathbf{k}} \sum_{\mathbf{k}_{1}} \widetilde{v}_{\mathbf{k}, t}^{x} G_{\mathbf{k},+}^{R} G_{\mathbf{k}_{1},-}^{R} G_{\mathbf{q}-\mathbf{k},-}^{R} G_{\mathbf{q}-\mathbf{k}_{1},+}^{R} \\
& \times \widetilde{v}_{\mathbf{q}-\mathbf{k}_{1},+}^{x} G_{\mathbf{q}-\mathbf{k}_{1},+}^{A} G_{\mathbf{k},+}^{A}\left\langle U_{\mathbf{k}, \mathbf{k}_{1}}^{+-} U_{\mathbf{q}-\mathbf{k}, \mathbf{q}-\mathbf{k}_{1}}^{-+}\right\rangle .
\end{aligned}
$$

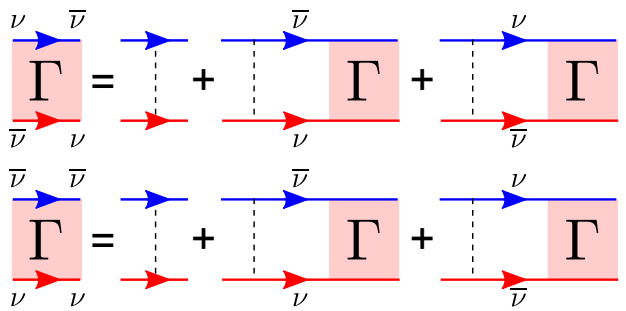

FIG. 9. (Color online) The Feynman diagrams of the BetheSalpeter equations for the intervalley cooperons. $v$ is the valley index, $v= \pm, \bar{v}=-$ if $v=+$.

In polar coordinates, the intervalley cooperons can be found from the coupled Bethe-Salpeter equations (see Fig. 9):

$$
\begin{aligned}
& \Gamma_{-+}^{+-}\left(\mathbf{k}_{1}, \mathbf{k}_{2}\right) \\
&= \gamma_{-+}^{+-}\left(\mathbf{k}_{1}, \mathbf{k}_{2}\right)+\int_{0}^{2 \pi} \frac{d \varphi}{2 \pi} \int_{0}^{\pi} \frac{d \theta \sin \theta}{2 \pi} \\
& \times \int_{0}^{\infty} \frac{d k k^{2}}{2 \pi} \sum_{\nu= \pm} \gamma_{-\bar{v}}^{+v}\left(\mathbf{k}_{1}, \mathbf{k}\right) \mathcal{G}_{\mathbf{k}}^{i \epsilon_{n}} \mathcal{G}_{\mathbf{q}-\mathbf{k}}^{i \epsilon_{n}-i \omega_{m}} \Gamma_{\bar{\nu}+}^{\nu-}\left(\mathbf{k}, \mathbf{k}_{2}\right), \\
& \Gamma_{++}^{--}\left(\mathbf{k}_{1}, \mathbf{k}_{2}\right) \\
&=\gamma_{++}^{--}\left(\mathbf{k}_{1}, \mathbf{k}_{2}\right)+\int_{0}^{2 \pi} \frac{d \varphi}{2 \pi} \int_{0}^{\pi} \frac{d \theta \sin \theta}{2 \pi} \\
& \quad \times \int_{0}^{\infty} \frac{d k k^{2}}{2 \pi} \sum_{\nu= \pm} \gamma_{+\bar{v}}^{-v}\left(\mathbf{k}_{1}, \mathbf{k}\right) \mathcal{G}_{\mathbf{k}}^{i \epsilon_{n}} \mathcal{G}_{\mathbf{q}-\mathbf{k}}^{i \epsilon_{n}-i \omega_{m}} \Gamma_{\bar{\nu}+}^{\nu-}\left(\mathbf{k}, \mathbf{k}_{2}\right),
\end{aligned}
$$

where $\bar{v}=-$ if $v=+$ and

$$
\begin{aligned}
\gamma_{\bar{v} v}^{v \bar{v}}\left(\mathbf{k}_{1}, \mathbf{k}_{2}\right) \equiv & \left\langle U_{\mathbf{k}_{1}, \mathbf{k}_{2}}^{\nu \bar{v}} U_{-\mathbf{k}_{1},-\mathbf{k}_{2}}^{\bar{\nu} v}\right\rangle \\
= & \frac{\hbar \eta_{I}}{2 \pi N_{F} \tau}\left[\frac{1}{2}\left(1+v \cos \theta_{1}\right)\left(1+\bar{v} \cos \theta_{2}\right)\right. \\
& -\sin \theta_{1} \sin \theta_{2} e^{i\left(\varphi_{2}-\varphi_{1}\right)} \\
& \left.+\frac{1}{2}\left(1+\bar{v} \cos \theta_{1}\right)\left(1+v \cos \theta_{2}\right) e^{i 2\left(\varphi_{2}-\varphi_{1}\right)}\right], \\
\gamma_{\overline{v v}}^{v v}\left(\mathbf{k}_{1}, \mathbf{k}_{2}\right) \equiv & \left\langle U_{\mathbf{k}_{1}, \mathbf{k}_{2}}^{v \nu} U_{-\mathbf{k}_{1},-\mathbf{k}_{2}}^{\overline{v v}}\right\rangle \\
= & \frac{\hbar \eta_{*}}{2 \pi N_{F} \tau}\left[\frac{1}{2}\left(1+v \cos \theta_{1}\right)\left(1+v \cos \theta_{2}\right)\right. \\
& +\sin \theta_{1} \sin \theta_{2} e^{i\left(\varphi_{2}-\varphi_{1}\right)} \\
& \left.+\frac{1}{2}\left(1+\bar{v} \cos \theta_{1}\right)\left(1+\bar{v} \cos \theta_{2}\right) e^{i 2\left(\varphi_{2}-\varphi_{1}\right)}\right],
\end{aligned}
$$

where $v= \pm, \eta_{I} \equiv \tau / \tau_{I}$, and $\eta_{*} \equiv \tau / \tau_{*}$. We assume

$$
\begin{aligned}
\Gamma_{-+}^{+-}\left(\mathbf{k}_{1}, \mathbf{k}_{2}\right)= & \frac{\hbar}{2 \pi N_{F} \tau}\left[a_{1}\left(1+\cos \theta_{1}\right)\left(1-\cos \theta_{2}\right)\right. \\
& -a_{2} \sin \theta_{1} \sin \theta_{2} e^{i\left(\varphi_{2}-\varphi_{1}\right)} \\
& \left.+a_{3}\left(1-\cos \theta_{1}\right)\left(1+\cos \theta_{2}\right) e^{i 2\left(\varphi_{2}-\varphi_{1}\right)}\right], \\
\Gamma_{++}^{--}\left(\mathbf{k}_{1}, \mathbf{k}_{2}\right)= & \frac{\hbar}{2 \pi N_{F} \tau}\left[b_{1}\left(1-\cos \theta_{1}\right)\left(1-\cos \theta_{2}\right)\right. \\
& +b_{2} \sin \theta_{1} \sin \theta_{2} e^{i\left(\varphi_{2}-\varphi_{1}\right)} \\
& \left.+b_{3}\left(1+\cos \theta_{1}\right)\left(1+\cos \theta_{2}\right) e^{i 2\left(\varphi_{2}-\varphi_{1}\right)}\right],
\end{aligned}
$$


and put them into the Bethe-Salpeter equations, we arrive at

$$
\begin{array}{ll}
a_{1}=a_{3}=\frac{1}{2 \ell^{2}} \frac{\chi_{1}^{a}}{Q_{1}^{2}+q^{2}}, & a_{2}=\frac{1}{\ell^{2}} \frac{\chi_{2}^{a}}{Q_{2}^{2}+q^{2}}, \\
b_{1}=b_{3}=\frac{1}{2 \ell^{2}} \frac{\chi_{1}^{b}}{Q_{1}^{2}+q^{2}}, \quad b_{2}=\frac{1}{\ell^{2}} \frac{\chi_{2}^{b}}{Q_{2}^{2}+q^{2}},
\end{array}
$$

where $q^{2}=q_{x}^{2}+q_{y}^{2}+q_{z}^{2}$ and other quantities are defined in Eq. (50).

\section{Conductivity and magnetoconductivity from quantum interference}

The total conductivity from the quantum interference has two parts

$$
\sigma^{q i}=2 \sigma_{0}^{q i}+\sigma_{I}^{q i}
$$

For a single valley,

$$
\sigma_{0}^{q i} \approx \frac{e^{2}}{h} \frac{2+\eta_{I}}{\left(1+\eta_{I}\right)^{2}} \sum_{\mathbf{q}} \frac{1}{Q_{0}^{2}+q^{2}},
$$

where

$$
Q_{0}^{2} \equiv \frac{\left(2+\eta_{I}\right) \eta_{I}}{2\left(1-\eta_{I}\right)} \frac{1}{\ell^{2}}
$$

and $\eta_{I}=\tau / \tau_{I}$ measures the weight of the intervalley scattering in the total scattering. The total scattering time $\tau$ is defined as $1 / \tau=1 / \tau_{0}+1 / \tau_{I}, \tau_{0}$ and $\tau_{I}$ are the intravalley and intervalley scattering times, respectively.

The intervalley part

$$
\sigma_{I}^{q i}=2\left(\sigma_{c 1}+2 \sigma_{c 2}+2 \sigma_{d 2}\right),
$$

where

$$
\begin{aligned}
& \sigma_{c 1}^{x}=-\frac{e^{2}}{h} \frac{1}{\left(1+\eta_{I}\right)^{2}} \sum_{\mathbf{q}}\left(\frac{18}{5} \frac{\chi_{1}^{a}}{Q_{1}^{2}+q^{2}}+\frac{12}{5} \frac{\chi_{2}^{a}}{Q_{2}^{2}+q^{2}}\right), \\
& \sigma_{c 2}^{x}=\frac{e^{2}}{h} \frac{1}{\left(1+\eta_{I}\right)^{2}} \sum_{\mathbf{q}} \frac{\eta_{*}}{2}\left(\frac{\chi_{1}^{a}}{Q_{1}^{2}+q^{2}}+\frac{\chi_{2}^{a}}{Q_{2}^{2}+q^{2}}\right), \\
& \sigma_{d 2}^{x}=-\frac{e^{2}}{h} \frac{1}{\left(1+\eta_{I}\right)^{2}} \sum_{\mathbf{q}} \frac{\eta_{I}}{2}\left(\frac{\chi_{1}^{b}}{Q_{1}^{2}+q^{2}}+\frac{\chi_{2}^{b}}{Q_{2}^{2}+q^{2}}\right),
\end{aligned}
$$

and $q^{2}=q_{x}^{2}+q_{y}^{2}+q_{z}^{2}$

$$
\begin{aligned}
Q_{1}^{2} & =\frac{\left(1-\frac{2}{3} \eta_{*}\right)^{2}-\left(\frac{2}{3} \eta_{I}\right)^{2}}{\varpi_{1} \ell^{2}}, \quad Q_{2}^{2}=\frac{\left(1-\frac{2}{3} \eta_{*}\right)^{2}-\left(\frac{2}{3} \eta_{I}\right)^{2}}{\varpi_{2} \ell^{2}}, \\
\chi_{1}^{a} & =\eta_{I} / \varpi_{1}, \quad \chi_{1}^{b}=\left[\eta_{*}+2\left(\eta_{I}^{2}-\eta_{*}^{2}\right) / 3\right] / \varpi_{2}, \\
\chi_{2}^{a} & =\eta_{I} / \varpi_{2}, \quad \chi_{2}^{b}=\left[\eta_{*}+2\left(\eta_{I}^{2}-\eta_{*}^{2}\right) / 3\right] / \varpi_{2}, \\
\varpi_{1} & =\frac{16}{15} \eta_{*}+\frac{22}{45}\left(\eta_{I}^{2}-\eta_{*}^{2}\right), \quad \varpi_{2}=\frac{8}{15} \eta_{*}+\frac{16}{45}\left(\eta_{I}^{2}-\eta_{*}^{2}\right), \\
\eta_{I} & =\tau / \tau_{I}, \quad \eta_{*}=\tau / \tau_{*} .
\end{aligned}
$$

One can check that $\sigma_{I}^{q i}$ vanishes when $\eta_{I}=0$.

To have the temperature dependence of the conductivity, one just replaces all the $\sum_{\mathbf{q}} \frac{1}{Q_{i}^{2}+q^{2}}$ in $\sigma^{q i}$ by

$$
\frac{1}{2 \pi^{2}} \int_{1 / \ell_{\phi}}^{1 / \ell} q^{2} d q
$$

The temperature dependence is contained in the phase coherence length $\ell_{\phi}=C / T^{p / 2}, C$ is a constant. In three dimensions, $p=3 / 2(p=3)$ if the electron-electron (electron-phonon) interaction is the decoherence mechanism [29].

To calculate the magnetoconductivity, one just replaces all the $\sum_{\mathbf{q}} \frac{1}{Q_{i}^{2}+q^{2}}$ in $\sigma^{q i}$ by

$$
\begin{aligned}
\Psi_{3}\left(B, Q_{i}\right)= & \int_{0}^{1 / \ell} \frac{d x}{(2 \pi)^{2}}\left[\psi\left(\frac{\ell_{B}^{2}}{\ell_{\phi}^{2}} \frac{\ell_{B}^{2}}{\ell^{2}}+\ell_{B}^{2}\left(Q_{i}^{2}+x^{2}\right)+\frac{1}{2}\right)\right. \\
& \left.-\psi\left(\frac{\ell_{B}^{2}}{\ell_{\phi}^{2}}+\ell_{B}^{2}\left(Q_{i}^{2}+x^{2}\right)+\frac{1}{2}\right)\right]
\end{aligned}
$$

where the magnetic length $\ell_{B} \equiv \sqrt{\hbar / 4 e B}$, the magnetic field $B$ is along arbitrary directions. The magnetoconductivity is defined as

$$
\delta \sigma^{q i}(B) \equiv \sigma^{q i}(B)-\sigma^{q i}(0) .
$$

\section{Conductivity and magnetoconductivity of a single valley of Weyl fermions}

For a single valley in absence of intervalley scattering, $\sigma^{q i}$ reduces to $\sigma_{0}^{q i}$ in Eq. (46) with $\eta_{I}=0$,

$$
\sigma^{q i}=\frac{e^{2}}{h} 2 \sum_{\mathbf{q}} \frac{1}{q^{2}} \text {. }
$$

Replace the summation by the integral in Eq. (51),

$$
\sigma^{q i}=\frac{e^{2}}{h} 2 \frac{1}{2 \pi^{2}} \int_{1 / \ell_{\phi}}^{1 / \ell} d q \frac{1}{q^{2}} q^{2}=\frac{e^{2}}{h} \frac{1}{\pi^{2}}\left(\frac{1}{\ell}-\frac{1}{\ell_{\phi}}\right) .
$$

This is of the same magnitude of Eq. (2.25a) of Ref. [29] but differs by a minus sign (note that the spin degeneracy 2 is included in Ref. [29]).

Replace the summation in Eq. (54) by Eq. (52), $\sigma^{q i}(B)$ of a single valley is found as

$$
\sigma^{q i}(B)=\frac{e^{2}}{h} 2 \Psi_{3}(B),
$$

and the magnetoconductivity is

$$
\delta \sigma^{q i}(B) \equiv \sigma^{q i}(B)-\sigma^{q i}(0),
$$

where

$$
\begin{aligned}
\sigma^{q i}(B)= & \frac{2 e^{2}}{h} \int_{0}^{1 / \ell} \frac{d x}{(2 \pi)^{2}}\left[\psi\left(\frac{\ell_{B}^{2}}{\ell_{\phi}^{2}} \frac{\ell_{B}^{2}}{\ell^{2}}+\ell_{B}^{2} x^{2}+\frac{1}{2}\right)\right. \\
& \left.-\psi\left(\frac{\ell_{B}^{2}}{\ell_{\phi}^{2}}+\ell_{B}^{2} x^{2}+\frac{1}{2}\right)\right],
\end{aligned}
$$

where the magnetic length $\ell_{B} \equiv \sqrt{\hbar / 4 e B}$, the magnetic field $B$ is along arbitrary directions, $\ell$ is the mean free path, and $\ell_{\phi}$ is the phase coherence length.

\section{CONDUCTIVITY CORRECTION FROM INTERACTION}

The leading-order of the self-energy from the interplay of interaction and disorder is the Fock (exchange) diagram 

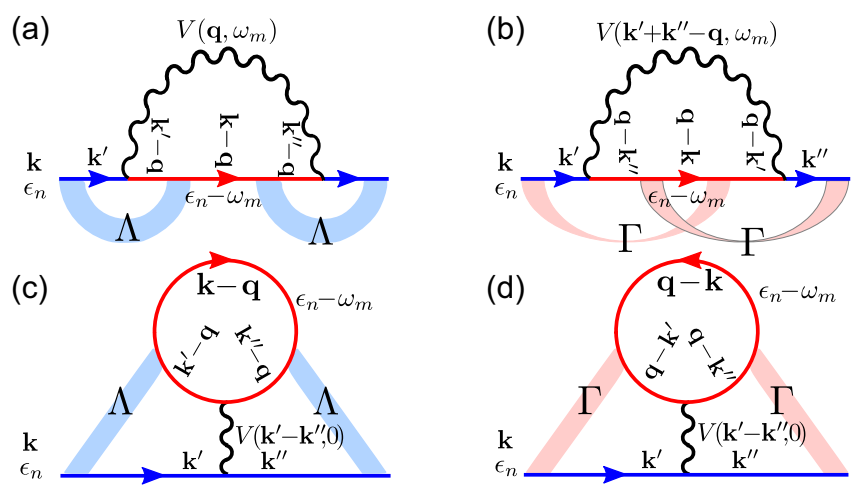

FIG. 10. (Color online) The Feynman diagrams for the selfenergies arising from the interplay of electron-electron interaction and disorder scattering [38,39].

dressed by diffusons [Fig. 10(a)],

$$
\begin{aligned}
\Sigma_{D}^{F}\left(\mathbf{k}, i \epsilon_{n}\right)= & -\frac{1}{\beta} \sum_{\omega_{m}} \sum_{\mathbf{q}} \mathcal{G}_{\mathbf{k}-\mathbf{q}}^{i \epsilon_{n}-i \omega_{m}} \sum_{\mathbf{k}^{\prime}} \sum_{\mathbf{k}^{\prime \prime}} V\left(\mathbf{q}, i \omega_{m}\right) \\
& \times \Lambda_{\mathbf{k}-\mathbf{q} \leftarrow \mathbf{k}^{\prime}, \epsilon_{n}}^{\mathbf{k} \leftarrow \mathbf{k}, i \epsilon_{n}-i \omega_{m}} \Lambda_{\mathbf{k}^{\prime \prime}-\mathbf{q}, \mathbf{q} \leftarrow \mathbf{k}-\mathbf{q}, i \epsilon_{n}-i \omega_{m}}^{\mathbf{k}^{\prime \prime}} \\
& \times \mathcal{G}_{\mathbf{k}^{\prime}}^{i \epsilon_{n}} \mathcal{G}_{\mathbf{k}^{\prime}-\mathbf{q}}^{i \epsilon_{n}-i \omega_{m}} \mathcal{G}_{\mathbf{k}^{\prime \prime}-\mathbf{q}}^{i \epsilon_{n}-i \omega_{m}} \mathcal{G}_{\mathbf{k}^{\prime \prime}}^{i \epsilon_{n}},
\end{aligned}
$$

where $1 / \beta=k_{B} T$, later we will show how to calculate the diffuson $\Lambda$ and interaction $V$. We find that (Sec. IX A)

$$
\begin{aligned}
\Lambda_{\mathbf{k}-\mathbf{q} \leftarrow \mathbf{k}^{\prime}-\mathbf{q}, i \epsilon_{n}-i \omega_{m}}^{\mathbf{k} \rightarrow \mathbf{k}^{\prime}, \epsilon_{n}} & =\Lambda_{\mathbf{k}^{\prime \prime}-\mathbf{q} \leftarrow \mathbf{k} \leftarrow \mathbf{k}-\mathbf{q}, i \epsilon_{n}-i \omega_{m}}^{\substack{\mathbf{k}^{\prime \prime} \rightarrow \mathbf{t}\\
}} \\
& =\Lambda_{\mathbf{k}_{1}, \mathbf{k}_{2}} \approx \frac{\hbar}{2 \pi N_{F} \tau^{2}} \frac{1}{\omega_{m}+D q^{2}},
\end{aligned}
$$

and

$$
\sum_{\mathbf{k}^{\prime}} \mathcal{G}_{\mathbf{k}^{\prime}}^{i \epsilon_{n}} \mathcal{G}_{\mathbf{k}^{\prime}-\mathbf{q}}^{i \epsilon_{n}-i \omega_{m}} \approx \frac{2 \pi N_{F} \tau}{\hbar} \theta\left[\epsilon_{n}\left(\omega_{m}-\epsilon_{n}\right)\right]
$$

Then

$$
\begin{aligned}
& \Sigma_{D}^{F}\left(\mathbf{k}, i \epsilon_{n}\right) \\
& \quad \approx-\frac{1}{\beta} \sum_{\omega_{m}} \mathcal{G}_{\mathbf{k}}^{i \epsilon_{n}-i \omega_{m}} \frac{1}{\tau^{2}} \sum_{\mathbf{q}} \frac{V_{\mathbf{q}}^{i \omega_{m}} \theta\left[\epsilon_{n}\left(\omega_{m}-\epsilon_{n}\right)\right]}{\left(\omega_{m}+D q^{2}\right)^{2}} .
\end{aligned}
$$

It is of the same form as Eq. (3.16) in Ref. [65]. Therefore the leading-order self-energy and its associated contribution to the conductivity have the same forms as those for the conventional electron with dispersion $p^{2} / 2 m$. The difference is that $D$ and $N_{F}$ need to be changed to those for the Weyl fermions.

\section{A. Diffuson}

In polar coordinates, the diffuson can be found from the Bethe-Salpeter equation (Fig. 11)

$$
\begin{aligned}
\Lambda_{\mathbf{k}_{1}, \mathbf{k}_{2}}= & \Lambda_{\mathbf{k}_{1}, \mathbf{k}_{2}}^{0}+\int_{0}^{2 \pi} \frac{d \varphi}{2 \pi} \int_{0}^{\pi} \frac{d \theta \sin \theta}{2 \pi} \int_{0}^{\infty} \frac{d k k^{2}}{2 \pi}, \\
& \times \Lambda_{\mathbf{k}_{1}, \mathbf{k}}^{0} \mathcal{G}_{\mathbf{k}}^{i \epsilon_{n}} \mathcal{G}_{\mathbf{k}-\mathbf{q}}^{i \epsilon_{n}-i \omega_{m}} \Lambda_{\mathbf{k}, \mathbf{k}_{2}},
\end{aligned}
$$

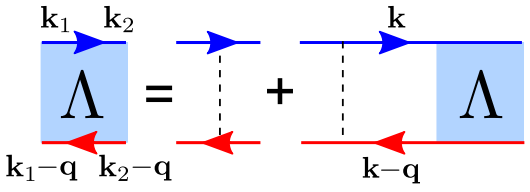

FIG. 11. (Color online) The Feynman diagram of the BetheSalpeter equation for the diffuson.

where the bare diffuson can be found:

$$
\begin{aligned}
\Lambda_{\mathbf{k}_{1}, \mathbf{k}_{2}}^{0} \equiv & \left\langle U_{\mathbf{k}_{1}, \mathbf{k}_{2}} U_{\mathbf{k}_{2}, \mathbf{k}_{1}}\right\rangle \\
\approx & \frac{\hbar}{2 \pi N_{F} \tau}\left[1+\left(1-2 \eta_{I}\right)\left(\cos \theta_{1} \cos \theta_{2}\right.\right. \\
& \left.\left.+\sin \theta_{1} \sin \theta_{2} \cos \left(\varphi_{1}-\varphi_{2}\right)\right)\right],
\end{aligned}
$$

and it can be found that

$$
\int_{0}^{\infty} \frac{d k k^{2}}{2 \pi} \mathcal{G}_{\mathbf{k}}^{i \epsilon_{n}} \mathcal{G}_{\mathbf{k}-\mathbf{q}}^{i \epsilon_{n}-i \omega_{m}}=\frac{2 \pi^{2} N_{F} \tau}{\hbar} \frac{1}{1+\omega_{m} \tau+i \tau \mathbf{v}_{F} \cdot \mathbf{q}}
$$

For convenience, the $z$ axis of $\mathbf{k}$ can be chosen to be along the direction of $\mathbf{q}$, then $\mathbf{v}_{F} \cdot \mathbf{q}=v_{F} q \cos \theta$ and

$$
\begin{aligned}
& \frac{1}{1+\omega_{m} \tau+i \tau \mathbf{v}_{F} \cdot \mathbf{q}} \\
& \quad \approx 1-\omega_{m} \tau-i \tau v_{F} q \cos \theta-\tau^{2} v_{F}^{2} q^{2} \cos ^{2} \theta .
\end{aligned}
$$

We assume the form of the full diffuson to be

$$
\begin{aligned}
\Lambda_{\mathbf{k}_{1}, \mathbf{k}_{2}} \approx & \frac{\hbar}{2 \pi N_{F} \tau}\left[d_{1}+d_{2} \cos \theta_{1}+d_{3} \cos \theta_{2}+d_{4} \cos \theta_{1} \cos \theta_{2}\right. \\
& \left.+d_{5} \sin \theta_{1} \sin \theta_{2} \cos \left(\varphi_{1}-\varphi_{2}\right)\right],
\end{aligned}
$$

with the coefficients $d_{i}$ to be determined. By putting it into the Bethe-Salpeter equation, we find that only the $d_{1}$ term is divergent as $q, \omega_{m} \rightarrow 0$ and

$$
\Lambda_{\mathbf{k}_{1}, \mathbf{k}_{2}} \approx \frac{\hbar}{2 \pi N_{F} \tau^{2}} \frac{1}{\omega_{m}+D q^{2}},
$$

where the diffusion coefficient

$$
D=\frac{1}{3} v_{F}^{2} \tau \eta_{v} .
$$

It is worth noting that here the expression of $D$ derived from the diffuson coincides with that in the semiclassical conductivity (Sec. VII). The above calculation does not distinguish interand intravalley scattering $\Lambda=\Lambda_{++}^{++}+\Lambda_{+-}^{+-}$.

\section{B. Interaction and random phase approximation}

After the Fourier transformation, the Hamiltonian of the interaction becomes

$$
V=\sum_{\mathbf{k}, \mathbf{k}^{\prime}, \mathbf{q}} \frac{V_{\mathbf{q}}}{2}\left(\phi_{\mathbf{k}}^{\dagger} \cdot \phi_{\mathbf{k}+\mathbf{q}}\right)\left(\phi_{\mathbf{k}^{\prime}}^{\dagger} \cdot \phi_{\mathbf{k}^{\prime}-\mathbf{q}}\right) c_{\mathbf{k}^{\prime}}^{\dagger} c_{\mathbf{k}}^{\dagger} c_{\mathbf{k}+\mathbf{q}} c_{\mathbf{k}^{\prime}-\mathbf{q}},
$$

where $\phi$ 's are the spinor wave functions, $c$ 's are corresponding operators, and

$$
V_{\mathbf{q}}=\int \mathrm{d}^{3} \mathbf{r} \frac{e^{2}}{4 \pi \varepsilon r} e^{-\mathrm{i} \mathbf{q} \cdot \mathbf{r}}=\frac{e^{2}}{\varepsilon q^{2}}
$$




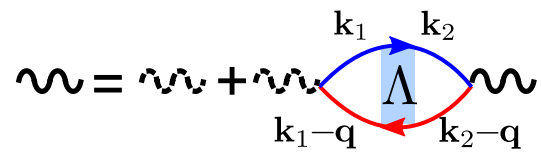

FIG. 12. (Color online) The Feynman diagram for the interaction (dashed wavy lines for bare interaction), which is renormalized (solid wavy lines) under random phase approximation. Different from in a clean system, the density function is dressed by the diffuson $(\Lambda)$.

with $\varepsilon$ the dielectric constant. Because $v_{q}$ diverges as $q \rightarrow 0$, then the spinor wave function part vanishes for a single band in the interaction potential

$$
V_{0}(q)=v_{\mathbf{q}}\left(\phi_{\mathbf{k}}^{\dagger} \cdot \phi_{\mathbf{k}+\mathbf{q}}\right)\left(\phi_{\mathbf{k}^{\prime}}^{\dagger} \cdot \phi_{\mathbf{k}^{\prime}-\mathbf{q}}\right) \approx V_{\mathbf{q}} .
$$

The long-range (bare) interaction is renormalized under the random phase approximation (Fig. 12):

$$
V\left(\mathbf{q}, \omega_{m}\right)=\frac{V_{0}(q)}{1+V_{0}(q) \Pi\left(q, \omega_{m}\right)},
$$

where, different from in a clean system, the density response function is dressed by the diffuson and takes the form

$$
\Pi\left(\mathbf{q}, \omega_{m}\right)=N_{F} \frac{D q^{2}}{\omega_{m}+D q^{2}} .
$$

Then

$$
V\left(\mathbf{q}, i \omega_{m}\right)=\frac{e^{2}}{\varepsilon q^{2}+e^{2} N_{F} \frac{D q^{2}}{\omega_{m}+D q^{2}}} .
$$

In the limit that $\omega_{m}, q \rightarrow 0$, the dynamically-screened interaction becomes

$$
V\left(\mathbf{q}, i \omega_{m}\right) \approx \frac{1}{N_{F}} \frac{\omega_{m}+D q^{2}}{D q^{2}} .
$$

This renormalized interaction is the one that is used in calculating the self-energy induced by the interplay of interaction and disorder.

\section{Screening factor}

The contribution from other three one-loop interaction diagrams [see Figs. 10(b)-10(d)] is proportional to the screening factor $F$, which is defined as

$$
F \equiv \frac{\left\langle V\left(\mathbf{k}-\mathbf{k}^{\prime}\right)\right\rangle_{k_{F}}}{V(0)},
$$

where $\langle\ldots\rangle_{k_{F}}$ means the average of the interaction $V\left(\mathbf{k}-\mathbf{k}^{\prime}\right)$ over momenta $\mathbf{k}$ and $\mathbf{k}^{\prime}$ on the Fermi surface.

In three dimensions [65],

$$
F=\frac{\ln \left[\left(1+\left(2 k_{F} \xi\right)^{2}\right]\right.}{\left(2 k_{F} \xi\right)^{2}},
$$

where $k_{F}$ the Fermi wave vector and $\xi$ is the screening length of the interaction,

$$
\xi^{2}=\frac{\varepsilon}{e^{2} N_{F}}
$$
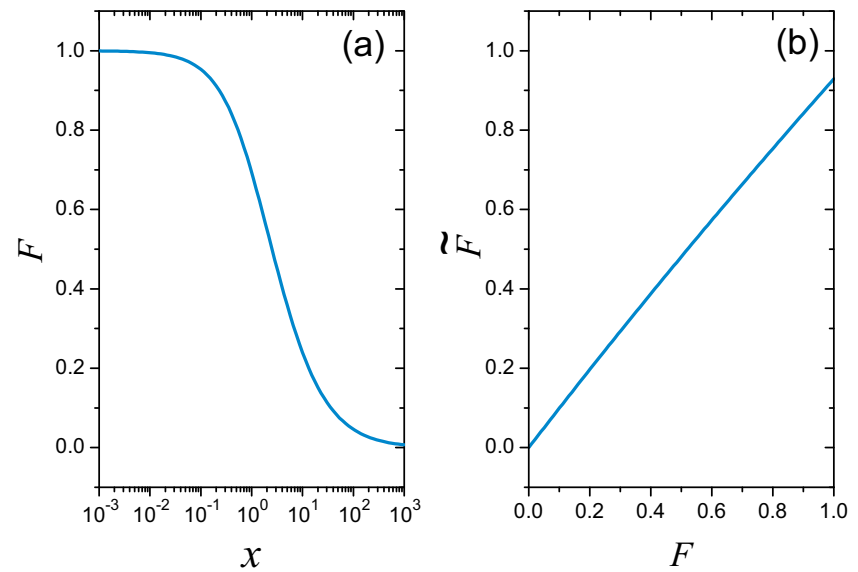

FIG. 13. (Color online) (a) The screening factor $F$ as a function of $x=8 \pi^{2} v_{F} \hbar \varepsilon / e^{2}$ for Weyl fermions. $v_{F}$ is the Fermi velocity, $\hbar$ is the reduced Planck's constant, and $\varepsilon$ is the dielectric constant. $-e$ is the electron charge. (b) In three dimensions, $\widetilde{F}$ as a function of $F . \tilde{F}$ is the renormalized screening factor after including the second-order diagrams and correction of the disorder by the interaction.

Using the density of states for the Weyl fermions $N_{F}=$ $E_{F}^{2} / 2 \pi^{2}\left(v_{F} \hbar\right)^{3}$ and $E_{F}=v_{F} \hbar k_{F}$, we define

$$
x \equiv\left(2 k_{F} \xi\right)^{2}=\frac{8 \pi^{2} v_{F} \hbar \varepsilon}{e^{2}} .
$$

Figure 13 shows $F$ as a function of $x$. By definition, $F \in[0,1]$.

The screening factor $F$ will be renormalized after including the second-order diagrams and correction of the disorder by the interaction. The renormalized screening factor in three dimensions is [65]

$$
\widetilde{F}=-\frac{32}{3}\left[1+\frac{3 F}{4}-\left(1+\frac{F}{2}\right)^{3 / 2}\right] F,
$$

which is shown in Fig. 13 as a function of $F$. One can see that $\widetilde{F} \approx F$ as $F \rightarrow 0$ and $\widetilde{F} \approx 0.93 F$ as $F \rightarrow 1$.

\section{CLASSICAL MAGNETOCONDUCTIVITY FROM LORENTZ FORCE}

The classical negative magnetoconductivity as a result of the cyclotron motion driven by Lorentz force in a perpendicular magnetic field $B$ can be found as [59]

$$
\delta \sigma^{C}(B)=-\sigma^{s c}(\mu B)^{2},
$$

where $\sigma^{s c}$ is given by Eq. (26) and $\mu$ is given by Eq. (84). We arrive at

$$
\delta \sigma^{C}(B)=-\frac{e^{2}}{h} \frac{\ell^{3} \sqrt{3} \eta_{v}^{3 / 2}}{16 \pi \ell_{B}^{4}}=-\frac{e^{2}}{h} \frac{9}{32 \sqrt{2} \pi\left(1+\eta_{I}\right)^{3 / 2}} \frac{\ell^{3}}{\ell_{B}^{4}},
$$

where $\ell_{B} \equiv \sqrt{\hbar / 4 e B}$.

The mobility of one valley of Weyl fermion is found as

$$
\mu=\frac{e v_{F} \tau \eta_{v}}{\hbar k_{F}}=\frac{e \ell \sqrt{3 \eta_{v}}}{\hbar k_{F}} \approx \frac{e \ell}{\hbar k_{F}},
$$

where the mean free path $\ell \equiv \sqrt{D \tau}=v_{F} \tau \sqrt{\eta_{v} / 3} \approx v_{F} \tau$. 
The relation between the mobility and mean free path is approximated as

$$
\ell \approx \frac{\mu \hbar k_{F}}{e} \approx 66 \mu k_{F},
$$

where $\ell$ in $\mathrm{nm}, \mu$ is in $\mathrm{cm}^{2} /(\mathrm{V} \mathrm{s})$, and $k_{F}$ in $\AA^{-1}$. For $k_{F}=0.03$ and $\mu=10^{4}$, the mean free path is about $20 \mu \mathrm{m}$.

\section{CONCLUSIONS}

In this work, we study the quantum transport properties of a two-valley Weyl semimetal. We employ the Feynman diagram techniques to calculate the conductivity in the presence of disorder and interaction. We derive three dominant parts of the conductivity (Fig. 1), including the semiclassical (Drude) conductivity, the correction from the quantum interference [weak (anti)localization], and the correction from the interplay of electron-electron interaction and disorder scattering (Altshuler-Aronov effect).

The quantum interference gives the main contribution to the magnetoconductivity. For a single valley of Weyl fermions, the low-temperature magnetoconductivity is proportional to $-\sqrt{B}$, where $B$ is the magnetic field applied along an arbitrary direction [see Fig. 3(a)]. This $-\sqrt{B}$ magnetoconductivity is from the weak antilocalization of Weyl fermions in the presence of weak intervalley scattering. Near zero field, the $-\sqrt{B}$ magnetoconductivity always overwhelms the positive $B^{2}$ magnetoconductivity from the chiral anomaly, giving another transport signature of Weyl semimetals. Strong intervalley scattering and correlation can lead to a crossover from the weak antilocalization to weak localization. During the crossover, the $-\sqrt{B}$ magnetoconductivity turns to $\sqrt{B}$ in the limit of strong intervalley scattering and correlation [see Fig. 3(c)]. By including the contributions from the weak antilocalization, Berry curvature correction, and Lorentz force (Table II), we compare the calculated magnetoconductivity with a recent experiment (see Fig. 4).

Both the quantum interference and interaction contribute to the temperature dependence of the conductivity. For a single valley of Weyl fermions, the weak antilocalization from the quantum interference gives a conductivity proportional to $-T^{p / 2}$, where $T$ is the temperature and the parameter $p$ is positive and depends on decoherence mechanisms. This conductivity thus always increases with decreasing temperature, giving another signature of the weak antilocalization. In contrast, the interaction gives a conductivity that decreases with decreasing temperature, following a $\sqrt{T}$ dependence. Therefore we expect a competition in the temperature dependence of the conductivity (see Fig. 2). Because $p$ is usually greater than 1 , the interaction always dominates below a critical temperature, leading to a tendency to localization in the temperature-dependent conductivity. We also present a systematic comparison of the transport properties for a single valley of Weyl fermions, 2D massless Dirac fermions, and 3D conventional electrons (Table III).

\section{ACKNOWLEDGMENTS}

This work was supported by Research Grants Council, University Grants Committee, Hong Kong, under Grant No. 17303714.
[1] L. Balents, Physics 4, 36 (2011).

[2] X. Wan, A. M. Turner, A. Vishwanath, and S. Y. Savrasov, Phys. Rev. B 83, 205101 (2011).

[3] K.-Y. Yang, Y.-M. Lu, and Y. Ran, Phys. Rev. B 84, 075129 (2011).

[4] A. A. Burkov and L. Balents, Phys. Rev. Lett. 107, 127205 (2011).

[5] P. Delplace, J. Li, and D. Carpentier, Europhys. Lett. 97, 67004 (2012).

[6] J.-H. Jiang, Phys. Rev. A 85, 033640 (2012).

[7] S. M. Young, S. Zaheer, J. C. Y. Teo, C. L. Kane, E. J. Mele, and A. M. Rappe, Phys. Rev. Lett. 108, 140405 (2012).

[8] G. Xu, H. Weng, Z. Wang, X. Dai, and Z. Fang, Phys. Rev. Lett. 107, 186806 (2011).

[9] Z. Wang, Y. Sun, X.-Q. Chen, C. Franchini, G. Xu, H. Weng, X. Dai, and Z. Fang, Phys. Rev. B 85, 195320 (2012).

[10] B. Singh, A. Sharma, H. Lin, M. Z. Hasan, R. Prasad, and A. Bansil, Phys. Rev. B 86, 115208 (2012).

[11] Z. Wang, H. Weng, Q. Wu, X. Dai, and Z. Fang, Phys. Rev. B 88, 125427 (2013).

[12] J. Liu and D. Vanderbilt, Phys. Rev. B 90, 155316 (2014).

[13] D. Bulmash, C.-X. Liu, and X.-L. Qi, Phys. Rev. B 89, 081106 (2014).

[14] M. Brahlek, N. Bansal, N. Koirala, S.-Y. Xu, M. Neupane, C. Liu, M. Z. Hasan, and S. Oh, Phys. Rev. Lett. 109, 186403 (2012).
[15] L. Wu, M. Brahlek, R. Valdes Aguilar, A. V. Stier, C. M. Morris, Y. Lubashevsky, L. S. Bilbro, N. Bansal, S. Oh, and N. P. Armitage, Nat. Phys. 9, 410 (2013).

[16] Z. K. Liu, B. Zhou, Y. Zhang, Z. J. Wang, H. M. Weng, D. Prabhakaran, S.-K. Mo, Z. X. Shen, Z. Fang, X. Dai, Z. Hussain, and Y. L. Chen, Science 343, 864 (2014).

[17] S.-Y. Xu, C. Liu, S. K. Kushwaha, R. Sankar, J. W. Krizan, I. Belopolski, M. Neupane, G. Bian, N. Alidoust, T.-R. Chang, H.-T. Jeng, C.-Y. Huang, W.-F. Tsai, H. Lin, P. P. Shibayev, F.-C. Chou, R. J. Cava, and M. Z. Hasan, Science 347, 294 (2015).

[18] Z. K. Liu, J. Jiang, B. Zhou, Z. J. Wang, Y. Zhang, H. M. Weng, D. Prabhakaran, S.-K. Mo, H. Peng, P. Dudin, T. Kim, M. Hoesch, Z. Fang, X. Dai, Z. X. Shen, D. L. Feng, Z. Hussain, and Y. L. Chen, Nat. Mater. 13, 677 (2014).

[19] M. Neupane, S.-Y. Xu, R. Sankar, N. Alidoust, G. Bian, C. Liu, I. Belopolski, T.-R. Chang, H.-T. Jeng, H. Lin, A. Bansil, F. Chou, and M. Z. Hasan, Nature Commun. 5, 3786 (2014).

[20] H. Yi, Z. Wang, C. Chen, Y. Shi, Y. Feng, A. Liang, Z. Xie, S. He, J. He, Y. Peng, X. Liu, Y. Liu, L. Zhao, G. Liu, X. Dong, J. Zhang, M. Nakatake, M. Arita, K. Shimada, H. Namatame, M. Taniguchi, Z. Xu, C. Chen, X. Dai, Z. Fang, and X. J. Zhou, Sci. Rep. 4, 6106 (2014).

[21] S. Borisenko, Q. Gibson, D. Evtushinsky, V. Zabolotnyy, B. Büchner, and R. J. Cava, Phys. Rev. Lett. 113, 027603 (2014). 
[22] S. Jeon, B. B. Zhou, A. Gyenis, B. E. Feldman, I. Kimchi, A. C. Potter, Q. D. Gibson, R. J. Cava, A. Vishwanath, and A. Yazdani, Nat. Mater. 13, 851 (2014).

[23] M. Novak, S. Sasaki, K. Segawa, and Y. Ando, Phys. Rev. B 91, 041203 (2015).

[24] P. Hosur, S. A. Parameswaran, and A. Vishwanath, Phys. Rev. Lett. 108, 046602 (2012).

[25] E. Fradkin, Phys. Rev. B 33, 3263 (1986).

[26] R. Shindou, R. Nakai, and S. Murakami, New J. Phys. 12, 065008 (2010).

[27] P. Goswami and S. Chakravarty, Phys. Rev. Lett. 107, 196803 (2011).

[28] S. V. Syzranov, L. Radzihovsky, and V. Gurarie, Phys. Rev. Lett. 114, 166601 (2015).

[29] P. A. Lee and T. V. Ramakrishnan, Rev. Mod. Phys. 57, 287 (1985).

[30] S. Hikami, A. I. Larkin, and Y. Nagaoka, Progr. Theor. Phys. 63, 707 (1980).

[31] H. Suzuura and T. Ando, Phys. Rev. Lett. 89, 266603 (2002).

[32] H.-J. Kim, K.-S. Kim, J.-F. Wang, M. Sasaki, N. Satoh, A. Ohnishi, M. Kitaura, M. Yang, and L. Li, Phys. Rev. Lett. 111, 246603 (2013).

[33] K.-S. Kim, H.-J. Kim, and M. Sasaki, Phys. Rev. B 89, 195137 (2014).

[34] Q. Li, D. E. Kharzeev, C. Zhang, Y. Huang, I. Pletikosic, A. V. Fedorov, R. D. Zhong, J. A. Schneeloch, G. D. Gu, and T. Valla, arXiv:1412.6543.

[35] S. M. Huang, S.-Y. Xu, I. Belopolski, C.-C. Lee, G. Chang, B. K. Wang, N. Alidoust, G. Bian, M. Neupane, C. Zhang, S. Jia, A. Bansil, H. Lin, and M. Z. Hasan, Nature Commun. 6, 7373 (2015).

[36] C. Zhang, S. Y. Xu, I. Belopolski, Z. Yuan, Z. Lin, B. Tong, N. Alidoust, C. C. Lee, S. M. Huang, H. Lin, M. Neupane, D. S. Sanchez, H. Zheng, G. Bian, J. Wang, C. Zhang, T. Neupert, M. Z. Hasan, and S. Jia, arXiv:1503.02630.

[37] E. McCann, K. Kechedzhi, V. I. Fal'ko, H. Suzuura, T. Ando, and B. L. Altshuler, Phys. Rev. Lett. 97, 146805 (2006).

[38] B. L. Altshuler, A. G. Aronov, and P. A. Lee, Phys. Rev. Lett. 44, 1288 (1980).

[39] H. Fukuyama, J. Phys. Soc. Jpn. 48, 2169 (1980).

[40] R. R. Biswas and S. Ryu, Phys. Rev. B 89, 014205 (2014).
[41] E. V. Gorbar, V. A. Miransky, and I. A. Shovkovy, Phys. Rev. B 89, 085126 (2014).

[42] I. Garate and L. Glazman, Phys. Rev. B 86, 035422 (2012).

[43] H.-Z. Lu, J. Shi, and S.-Q. Shen, Phys. Rev. Lett. 107, 076801 (2011).

[44] W.-Y. Shan, H.-Z. Lu, and S.-Q. Shen, Phys. Rev. B 86, 125303 (2012).

[45] H.-Z. Lu and S.-Q. Shen, Phys. Rev. Lett. 112, 146601 (2014).

[46] F. J. Dyson, J. Math. Phys. 3, 140 (1962).

[47] D. J. Thouless, Phys. Rev. Lett. 39, 1167 (1977).

[48] H.-Z. Lu and S.-Q. Shen, Phys. Rev. B 84, 125138 (2011).

[49] J. González, Phys. Rev. B 90, 121107 (2014).

[50] W. S. Boyle and A. D. Brailsford, Phys. Rev. 120, 1943 (1960).

[51] S. Golin, Phys. Rev. 166, 643 (1968).

[52] Y. Liu and R. E. Allen, Phys. Rev. B 52, 1566 (1995).

[53] J. Cisowski and J. Bodnar, J. Phys. Status Solidi (a) 28, K49 (1975).

[54] J.-P. Jay-Gerin, M. Aubin, and L. Caron, Solid State Commun. 21, 771 (1977).

[55] F. A. P. Blom and M. J. Gelten, Proceedings 3rd International Conference Physics of Narrow-gap Semiconductors, PWN Warsaw, 1977 (Elsevier, 1977), p. 257.

[56] T. Liang, Q. Gibson, M. N. Ali, M. Liu, R. J. Cava, and N. P. Ong, Nat. Mater. 14, 280 (2015).

[57] D. T. Son and B. Z. Spivak, Phys. Rev. B 88, 104412 (2013).

[58] A. A. Burkov, Phys. Rev. Lett. 113, 247203 (2014).

[59] S. Datta, Electronic Transport in Mesoscopic Systems (Cambridge University Press, 1997).

[60] J. Wang, H. Li, C. Chang, K. He, J. Lee, H. Lu, Y. Sun, X. Ma, N. Samarth, S. Shen, Q. Xue, M. Xie, and M. Chan, Nano Res. 5, 739 (2012).

[61] N. H. Shon and T. Ando, J. Phys. Soc. Jpn. 67, 2421 (1998).

[62] A. Kawabata, J. Phys. Soc. Jpn. 49, 628 (1980).

[63] H.-C. Liu, H.-Z. Lu, H.-T. He, B. Li, S.-G. Liu, Q. L. He, G. Wang, I. K. Sou, S.-Q. Shen, and J. Wang, ACS Nano 8, 9616 (2014).

[64] H.-Z. Lu, W. Yao, D. Xiao, and S.-Q. Shen, Phys. Rev. Lett. 110, 016806 (2013).

[65] B. L. Altshuler and A. G. Aronov, Electron-Electron Interactions in Disordered Systems (North-Holland, Amsterdam, 1985). 